\title{
$5 \quad$ Wer bildet die Lehrpersonen aus?
}

Sibylle Steinmann, Samuel Krattenmacher und Horst Biedermann

\begin{abstract}
Das vorliegende Kapitel beschäftigt sich mit den Dozierenden der Mathematik/Mathematikdidaktik und den Dozierenden der Erziehungswissenschaft an den Lehrerausbildungsinstitutionen. Nebst den demographischen Merkmalen der Lehrerausbildenden im nationalen und internationalen Vergleich werden die formalen Ausbildungsqualifikationen (akademische Vorbildung und schulische Lehrbefähigung), der Erfahrungshintergrund (Lehr- und Forschungserfahrung) und das vielfältige Tätigkeitsprofil der Dozierenden dargestellt. Die Resultate werden im Rahmen der Umsetzung des Verwissenschaftlichungsprozesses im Zuge der Tertiarisierung der deutschschweizerischen Lehrerausbildung diskutiert.
\end{abstract}

\subsection{Wer soll Lehrpersonen ausbilden?}

Lehrerausbildende haben die Aufgabe, angehende Lehrpersonen möglichst gut auf ihren Beruf vorzubereiten. Welche Kompetenzen, Qualifikationen und Erfahrungen Lehrerausbildende benötigen, um diese anspruchsvolle Aufgabe wahrzunehmen, ist indes noch nicht geklärt. Die Diskussion über die Art der notwendigen Ausbildung bewegt sich zwischen den Polen Praxisnähe und Akademisierung (Darling-Hammond, 2000).

Sind es die praktischen Erfahrungen als Lehrperson, welche Lehrerausbildende in besonderer Weise befähigen? Die Forderung nach Praktikerinnen und Praktikern in der Lehrerausbildung beruht auf der Annahme, dass gute Lehrpersonen auch gute Lehrerausbildende seien (Korthagen, Loughran \& Lunenberg, 2005). Wer das Feld kennt, kann praxisrelevante, umsetzbare, gar rezeptähnliche Anleitungen geben, weiss, wie eine Klasse $\mathrm{zu}$ managen ist, herausfordernde Aufgaben gestellt und erklärt werden können und wie Elternarbeit zu organisieren ist.

Oder ist es die akademische Qualifikation, welche Lehrerausbildende professionalisiert? Beispielsweise für die Vermittlung von fundiertem Professionswissen oder für das Anleiten zum Reflektieren über die Praxis als reflectiv 
practitioner (Schön, 1983) ist ein vertieftes, klares und explizites Verständnis des Lehrens und Lernens unumgänglich (Berry \& Loughran, 2002; Robinson \& McMillan, 2006). Die Gefahr liege jedoch in der Verakademisierung der Lehrerbildung. Wenn Lehrerausbildende durch ihre Praxisferne unumsetzbare Theorien vermitteln und nicht auf die Realität „Schule“ vorbereiten, scheitern die angehenden Lehrpersonen im Beruf (Furger, 2010). Ungeklärt ist auch, inwiefern sich Lehrerausbildende überhaupt für wissenschaftlich fundiertes Wissen (Hügli, 1998; Lunenberg et al., 2007) oder gar für eigene Forschungstätigkeit (bspw. Koster, Brekelmans, Korthagen \& Wubbels, 2005) interessieren und interessieren sollten.

Die Antwort auf die Frage nach der geeigneten Qualifikation der Lehrerausbildenden kann kaum im „Entweder-oder“, sondern nur im „Sowohl-alsauch" gefunden werden. Die Lehrerausbildung besteht aus einer Vielzahl von Fächern, aufgeteilt in Module und unterrichtet von unterschiedlichen Lehrerausbildenden. Nebst dem Studium des Faches (content knowledge ${ }^{1}$ ), lernen die angehenden Lehrpersonen auch, wie Inhalte vermittelt werden sollen (pedagogical content knowledge ${ }^{1}$ ) und was allgemein pädagogische und pädagogischpsychologische Theorien beinhalten (pedagogical knowledge ${ }^{1}$ ). In Praktika und anschliessenden Besprechungen reflektieren die Studierenden ihre Erfahrungen und bringen diese in Bezug zur Theorie. Diese verschiedenen Ausbildungsbereiche orientieren sich unterschiedlich stark an Theorie und Praxis und verlangen somit ein unterschiedliches Lehrerausbildungsprofil.

Beachtet werden muss ebenfalls, dass die Tätigkeitsfelder der Lehrerausbildenden in deutschsprachigen Ländern neben dem Ausbilden von Lehrpersonen zusätzlich wissenschaftliches Arbeiten und Forschen, Schaffen von Rahmenbedingungen für Lehren und Lernen und das Repräsentieren und Gestalten der Hochschule umfassen (bspw. Blömeke, Hascher \& Mayr, 2005). Die passende Qualifikation der Lehrerausbildenden muss demnach auch in Abhängigkeit von ihren Tätigkeitsfeldern und ihren Unterrichtsbereichen diskutiert werden.

In der Literatur und der Öffentlichkeit wird die Frage nach der Qualifikation der Lehrerausbildenden kontrovers zwischen den Polen der Praxis und der Akademisierung diskutiert. Die hier vorliegende Studie stellt die Lehrerausbildenden der Deutschschweiz im internationalen und deutschschweizerischen Kontext dar und geht der Frage nach ihren formalen Ausbildungsqualifikationen (akademischer Hintergrund und schulische Lehrbefähigung), ihrem Erfahrungshintergrund (Lehr- und Forschungserfahrung) und ihren Tätigkeiten an den Ausbildungsinstitutionen nach.

1 Entsprechend den drei Wissensbereichen (CK, PCK und PK) nach Shulman (1986). 


\subsection{Forschungsstand}

Trotz der unbestrittenen Bedeutung der Lehrerausbildenden wird ihnen in den meisten bisherigen Forschungsarbeiten, falls überhaupt, meist eine untergeordnete Rolle zugeschrieben (Lanier \& Little, 1986; Loughran, 1997; Robinson \& McMillan, 2006).

$\mathrm{Zu}$ den ersten empirischen Arbeiten im deutschen Sprachgebiet gehört der Beitrag von Criblez (2001) im Rahmen des Schweizerischen Nationalen Forschungsprogramms 33 „Die Wirksamkeit der Lehrerbildungssysteme“ von Oser und Oelkers (2001). Criblez gelang eine Charakterisierung der verschiedenen Ausbildenden für die Kindergarten- und Primarstufe sowie die Sekundarstufen I und II in der deutschsprachigen Schweiz. Die Studie wurde jedoch vor der Umsetzung der Reform der schweizerischen Lehrerbildung ${ }^{2}$ durchgeführt und dürfte somit nicht mehr den aktuellen Gegebenheiten entsprechen.

Die im Rahmen der MT21-Untersuchung (Blömeke, Kaiser \& Lehmann, 2008) durchgeführte Studie der Lehrerausbildenden von Felbrich, Müller und Blömeke (2008) gilt als die erste systematisch angelegte Studie mit internationalen Vergleichsdaten, gefolgt von der TEDS-M Studie und ersten Auswertungen dazu (siehe Felbrich, Schmotz, Kaiser, Hacke \& Lehmann, 2010; Schmotz, Felbrich, Lehmann, Hacke \& Kaiser, 2010). Die TEDS-M ist die erste internationale Vergleichsstudie mit repräsentativen Stichproben im Bereich der Lehrerausbildenden, welche auch für die Deutschschweiz eine gute Datenbasis liefert.

Über die Qualifikation und die Professionalität von Lehrerausbildenden war bis vor kurzem kaum etwas bekannt (bspw. Koster \& Dengerink, 2001). In den letzten Jahren entstanden vorwiegend deskriptive Arbeiten über den IstZustand der formalen Qualifikation von Lehrerausbildenden und ihren Aufgabenfeldern (Blömeke et al., 2005; Criblez, 2001; Felbrich et al., 2010; Lehmann, Criblez, Guldimann, Fuchs \& Périsset Bagnoud, 2007; Noel, 2006; Robinson \& McMillan, 2006; Schmotz et al., 2010; Smith, 2005). In wenigen Arbeiten werden Standards für Lehrerausbildende präsentiert und diskutiert (Koster et al., 2005; Koster \& Dengerink, 2001; Murray, 2001), wobei deutlich wird, dass nur ein kleiner Konsens bezüglich der Standards besteht (Murray, 2001). Empirische Untersuchungen über die Bedeutsamkeit der Professionalität und Qualifikation der Lehrerausbildenden fehlen unserem Wissen nach noch gänzlich.

In der Schweiz ist die Lehrerausbildung und somit auch die Situation für die Lehrerausbildenden in den vergangenen Jahren von der Reform der Lehrerbildung und der daraus folgenden Tertiarisierung geprägt: „Der Übergang vom Lehrerseminar zur Pädagogischen Hochschule (PH) ist mit einem ver-

2 Die Reform der schweizerischen Lehrerbildung hatte die Tertiarisierung der Lehrerbildung zum Ziel. Einen guten Überblick über die Reform erhält man in Criblez (2010). 
stärkten Wissenschafts- und Forschungsbezug in der Ausbildung der Lehrpersonen sowie mit der Erweiterung des Leistungsauftrags der Pädagogischen Hochschulen“ (EDK, 2007, S. 3) verbunden. Entsprechend den neuen Anforderungen an die Ausbildung musste, wie dies Criblez festhält, ,über die Qualifizierung von Ausbildnerinnen und Ausbildnern neu nachgedacht werden“ (2001, S. 478). 1999 hat die Schweizerische Konferenz der kantonalen Erziehungsdirektoren (EDK) Reglemente über die Qualifizierung von Lehrerausbildenden erlassen (EDK, 1999a; EDK, 1999b) und im Anschlussbericht zum Masterplan Pädagogische Hochschule (EDK, 2008) ergänzt und präzisiert. Die Schweizerische Konferenz der Rektorinnen und Rektoren der Pädagogischen Hochschulen (COHEP) nennt in ihrem Papier Strategien und Ziele für die Weiterentwicklungen (COHEP, 2007) und bei der Bilanztagung im Januar 2008 wurden Thesen formuliert (Ambühl \& Stadelmann, 2010). So sollen die Dozierenden der Pädagogischen Hochschulen ,auf hohem Niveau wissenschaftlich, fachlich und fachdidaktisch qualifiziert" (COHEP, 2007, S. 2) sein und u.a. nebst der Haupttätigkeit der Lehre auch aktiv forschen (Vogt \& Pilloud, 2010).

$\mathrm{Zu}$ den Lehrerausbildenden der Deutschschweiz liegen bisher exemplarische Daten zu vier Pädagogischen Hochschulen vor (siehe Lehmann et al., 2007), welche jedoch nicht als repräsentativ angesehen werden können. Weitere Daten zur Qualifizierung der Dozierenden finden sich im Anschlussbericht zum Masterplan Pädagogische Hochschule (EDK, 2008) und im Bildungsbericht Schweiz 2010 (Annen et al., 2010).

Aufgrund des Forschungsstandes, der spezifischen Situation der Deutschschweiz und der Annahme, dass gute Lehrerausbildende über spezifische Qualifikationen verfügen müssen, postulieren wir folgende Vermutungen, welche im vorliegenden Artikel überprüft werden sollen:

a) Die Deutschschweizer Dozierenden weisen im internationalen Vergleich eine mittlere akademische Grundbildung auf, da erst seit der kürzlich erfolgten Tertiarisierung der Lehrerausbildung explizit ein akademischer Abschluss der Dozierenden eingefordert wird (siehe Kapitel 5.4.2).

b) Trotz der Tertiarisierung und entsprechend dem Anforderungsprofil der EDK besitzt eine Mehrheit der Deutschschweizer Dozierenden ein Lehrdiplom für die Unterrichtszielstufe ihrer Studierenden (siehe Kapitel 5.4.3).

c) Es lässt sich nur eine Minderheit finden, die sich spezifisch auf die Aufgabe als Lehrerausbildende vorbereitet fühlt. Dies ist als Folge davon zu sehen, dass in der Deutschschweiz keine spezifische Ausbildung für Lehrerausbildende existiert (siehe Kapitel 5.4.4).

d) Durch den mit der Tertiarisierung verbundenen Forschungsauftrag an die Pädagogischen Hochschulen ist auch die Forschungserfahrung der Deutsch- 
schweizer Dozierenden an den Hochschulen deutlich ausgeprägt und es lassen sich diesbezüglich Mehrheiten finden (siehe Kapitel 5.4.6).

e) Durch die grössere Forschungserfahrung büssen die Deutschschweizer Dozierenden an Erfahrung auf der Zielstufe ein und es lässt sich daher keine diesbezügliche Mehrheit finden (siehe Kapitel 5.4.5).

f) Das Tätigkeitsprofil der Dozierenden setzt sich vor allem aus Teilen der Lehre und der Forschung zusammen (siehe Kapitel 5.4.7).

\subsection{Stichprobe der Dozierenden}

\subsubsection{Internationale Stichprobe}

In der Studie TEDS-M werden als Lehrerausbildende die Personen berücksichtigt, welche regelmässig und wiederkehrend direkt an Lehrerausbildungsinstitutionen unterrichten (im Folgenden als Dozierende bezeichnet). Die Praxislehrpersonen, welche ebenfalls in der Ausbildung in der Deutschschweiz tätig sind, gehören nicht zur internationalen Stichprobe. ${ }^{3}$

Die Dozierenden lassen sich einerseits nach dem unterrichteten Fach und andererseits nach der Unterrichtszielstufe ihrer Studierenden kategorisieren. International werden die Dozierenden nach ihrer Fachrichtung in die Gruppen der „Erziehungswissenschaft", „Mathematik/Mathematikdidaktik“ oder „Erziehungswissenschaft und gleichzeitig Mathematik/Mathematikdidaktik" eingeteilt. Für die Analyse der internationalen Daten berücksichtigen wir die Einteilung nach der Unterrichtszielstufe der angehenden Lehrpersonen. Um eine möglichst präzise Zuteilung zu generieren, werden für die folgenden Analysen Daten aus dem Dozierenden- und dem Institutsfragebogen ${ }^{4}$ verwendet. Im Fragebogen geben die Dozierenden an, wie lange sie bereits Studierende mit Zielstufe Primar- und/oder Sekundarstufe lehren. Mittels der Antworten können drei Gruppen gebildet werden: Dozierende für (a) ausschliesslich angehende Primarlehrpersonen, (b) Dozierende, welche Studierende für die Primar- und Sekundarstufe und für (c) ausschliesslich die Sekundarstufe unterrichten. Analog zum Untersuchungsdesign von TEDS-M sind die Dozierenden einer oder mehreren Institutionen zugeordnet. Die Institutionen werden gemäss den Definitionen von TEDS-M ebenfalls den drei oben beschriebenen Gruppen zugewiesen, was wiederum den Rückschluss auf die Zuordnung der Dozierenden

3 Die Praxislehrpersonen der Deutschschweiz wurden in einer Zusatzstudie befragt. Die Resultate dieser Zusatzstudie werden später veröffentlicht.

4 Die Institutsleitungspersonen wurden anhand eines Fragebogens zu ihrer Institution befragt. Mit diesem Institutsfragebogen wurden Angaben zu Richtlinien, dem Ausbildungsprogramm, Angaben zur Studierendenanzahl usw. erhoben (siehe auch Kapitel 2 in diesem Band). 
zulässt. Die Tabelle 5.1 zeigt auf, wie sich die Stichprobe in den Ländern entsprechend der drei Gruppen zusammensetzt. Norwegen, Kanada und die USA fallen aufgrund des zu kleinen Rücklaufs aus der internationalen Stichprobe heraus.

Die internationale Gesamtstichprobe umfasst total 5'184 Dozierenden, welche über $14^{`} 000$ Dozierende in den 14 Ländern repräsentieren. In zehn Ländern finden sich Dozierende, welche ausschliesslich angehende Primarstufenlehrpersonen ausbilden ( $\mathrm{N}=1$ '510). In elf Ländern gibt es insgesamt 1'419 Dozierende, welche sich ausschliesslich der Ausbildung angehender Sekundarstufenlehrpersonen widmen. Der grösste Anteil der Dozierenden ( $\mathrm{N}=2255)$ unterrichtet Studierende beider Zielstufen.

Tabelle 5.1: Internationale Stichprobe der Dozierenden nach den Zielstufen

\begin{tabular}{|c|c|c|c|c|c|c|c|c|c|}
\hline & \multicolumn{3}{|c|}{$\begin{array}{l}\text { Dozierende } \\
\text { ausschliesslich für } \\
\text { die Primarstufe }\end{array}$} & \multicolumn{3}{|c|}{$\begin{array}{l}\text { Dozierende, welche } \\
\text { auf beiden Stufen } \\
\text { lehren }\end{array}$} & \multicolumn{3}{|c|}{$\begin{array}{l}\text { Dozierende } \\
\text { ausschliesslich für } \\
\text { die Sekundarstufe }\end{array}$} \\
\hline & $\mathrm{N}$ & $\begin{array}{l}\text { Popu- } \\
\text { lation }\end{array}$ & $\%$ & $\mathrm{~N}$ & $\begin{array}{l}\text { Popu- } \\
\text { lation }\end{array}$ & $\%$ & $\mathrm{~N}$ & $\begin{array}{l}\text { Popu- } \\
\text { lation }\end{array}$ & $\%$ \\
\hline Botswana & 20 & 20 & 45.5 & 10 & 11 & 25.0 & 13 & 13 & 29.6 \\
\hline Chile $^{1}$ & 0 & & 0 & 387 & 721 & 98.9 & 5 & 8 & 1.1 \\
\hline Deutschland ${ }^{1}$ & 43 & 332 & 8.5 & 243 & 1265 & 32.4 & 194 & 2308 & 59.1 \\
\hline Deutschschweiz ${ }^{1}$ & 88 & 178 & 42.9 & 98 & 179 & 43.1 & 34 & 58 & 14.0 \\
\hline Georgien & 14 & 14 & 21.9 & 26 & 27 & 42.7 & 22 & 23 & 35.4 \\
\hline Malaysia $^{1}$ & 104 & 172 & 37.6 & 121 & 225 & 49.2 & 30 & 60 & 13.2 \\
\hline Oman & - & & - & - & & - & 84 & 103 & 100 \\
\hline Philippinen & 84 & 437 & 15.3 & 343 & 1705 & 59.9 & 162 & 706 & 24.8 \\
\hline Polen $^{2}$ & 307 & 437 & 37.3 & 423 & 737 & 62.8 & - & & - \\
\hline Russland & 225 & 652 & 20.8 & 176 & 519 & 16.6 & 811 & 1964 & 62.7 \\
\hline Singapur ${ }^{3}$ & - & & - & 73 & 86 & 94.7 & 4 & 5 & 5.3 \\
\hline Spanien & 533 & 770 & 100 & - & & - & - & & - \\
\hline Taiwan & 92 & 108 & 31.8 & 43 & 122 & 35.9 & 60 & 109 & 32.3 \\
\hline Thailand & - & & - & 312 & 354 & 100 & - & & - \\
\hline Total & 1510 & 3121 & 29.1 & 2255 & 5950 & 43.5 & 1419 & 5357 & 27.4 \\
\hline
\end{tabular}

Legende: N: Anzahl der Dozierenden, die an der Befragung teilgenommen haben

Population: Anzahl der Dozierenden, die in den einzelnen Ländern in den einzelnen Stufen unterrichten

\%: gibt an, wie gross die Teilgruppe der Dozierenden innerhalb eines Landes ist

Anmerkungen: ${ }^{1}$ kombinierte Rücklaufquote $<60 \%,{ }^{2}$ kombinierte Rücklaufquote $<75 \%$,

${ }^{3}$ Aufgrund der kleinen Fallzahl bei den Dozierenden der Sekundarstufe werden die

Teilstichproben in den weiteren Darstellungen weggelassen.

Wenn innerhalb einer Institution der Rücklauf < 50\% betrug, wurden die Dozierenden nicht in die Stichprobe aufgenommen. 


\subsubsection{Deutschschweizer Stichprobe}

Wie bereits erwähnt, unterscheiden sich die Dozierenden einerseits durch ihre Fachrichtungen (Mathematik/Mathematikdidaktik oder Erziehungswissenschaft $^{5}$ ) und andererseits durch die Zielstufe der von ihnen unterrichteten Studierenden (Primarschule, Sekundarstufe, beides). Für eine differenziertere Analyse der Dozierenden in der Deutschschweiz werden in den folgenden Kapiteln, anders als im internationalen Vergleich, beide Dimensionen berücksichtigt, so dass sich sechs Untergruppen ergeben (siehe Tabelle 5.2).

Die Dozierenden der Erziehungswissenschaft stellen mit 168 Dozierenden die deutlich grössere Gruppe als die der Mathematik/Mathematikdidaktik mit 52 Dozierenden dar. Innerhalb der Erziehungswissenschaft unterrichten 65 Dozierende ausschliesslich angehende Primarlehrpersonen, 18 nur angehende Sekundarstufenlehrpersonen und 85 Dozierende unterrichten Studierende beider Zielstufen. Bei den Dozierenden der Fachrichtung Mathematik/Mathematikdidaktik unterrichtet der kleinste Anteil Studierende beider Zielstufen $(\mathrm{N}=13), 16$ Personen unterrichten ausschliesslich angehende Sekundarstufenlehrpersonen und 23 Dozierende einzig angehende Primarlehrpersonen.

Tabelle 5.2: Stichprobe der Deutschschweizer Dozierenden differenziert nach Fachrichtung und Zielstufe der Studierenden

\begin{tabular}{lllll}
\cline { 2 - 5 } Fach & Zielstufe & N & Population & $\%$ \\
\hline \multirow{3}{*}{ Erziehungswissenschaft } & ausschliesslich Primarstufe & 65 & 144 & 34.6 \\
\cline { 2 - 5 } & Primar- und Sekundarstufe & 85 & 161 & 38.6 \\
\cline { 2 - 5 } & ausschliesslich Sekundarstufe & 18 & 34 & 8.1 \\
\hline \multirow{3}{*}{$\begin{array}{l}\text { Mathematik/ } \\
\text { Mathematikdidaktik }\end{array}$} & ausschliesslich Primarstufe & 23 & 35 & 8.3 \\
\cline { 2 - 5 } & Primar- und Sekundarstufe & 13 & 19 & 4.5 \\
\cline { 2 - 5 } & ausschliesslich Sekundarstufe & 16 & 24 & 5.9 \\
\hline Total & Total Dozierende CHD & 220 & 417 & 100 \\
\cline { 2 - 5 }
\end{tabular}

Legende: N: Anzahl der Dozierenden, die an der Befragung teilgenommen haben Population: Anzahl der Dozierenden, die in den einzelnen Ländern in den einzelnen Stufen unterrichten

\%: gibt an, wie gross die Teilgruppe der Dozierenden innerhalb der Deutschschweiz ist

5 In der Stichprobe der Deutschschweiz unterrichtet nur eine Person sowohl Mathematik/ Mathematikdidaktik als auch Erziehungswissenschaft. Diese Person wird in der hier vorliegenden Auswertung der Gruppe Mathematik/Mathematikdidaktik zugeordnet. 


\subsection{Ausgewählte Merkmale der Dozierenden}

\subsubsection{Geschlechtsspezifische Verteilung der Dozierenden}

Betrachtet man die Verteilung des Geschlechts, fällt auf, dass der Anteil an männlichen Dozierenden zwischen den Ländern und den Zielstufen zwischen dem Minimum von 5 (Russland, Zielstufe Primarstufe) und dem Maximum von 95\% (Oman, Zielstufe Sekundarstufe) erheblich variiert. Über alle Länder hinweg gesehen, ist der Anteil der männlichen Dozierenden auf der Zielstufe Sekundarstufe tendenziell grösser als auf der Zielstufe Primarstufe (Abbildung 5.2).

In der Deutschschweiz sind in allen drei Gruppen mehr als die Hälfte der Dozierenden männlich. Auch im internationalen Vergleich gesehen weist die Deutschschweiz einen hohen Anteil an männlichen Dozierenden auf. Bei der Zielstufe Primarstufe ist der Anteil der weiblichen Dozierenden in der Deutschschweiz mit nur 43\% sogar signifikant kleiner als der in Georgien, Polen, Deutschland und Russland, bei der Gruppe mit beiden Zielstufen ist der Anteil mit 35\% signifikant kleiner als in Chile, Thailand, Singapur, den Philippinen und Russland. Wegen der grossen Standardfehler unterscheiden sich Deutschland und Georgien nicht signifikant von der Deutschschweiz. Bei den Dozierenden der Primarstufe und den Dozierenden beider Stufen hat kein Land einen signifikant höheren Anteil an männlichen Dozierenden als die Deutschschweiz.

In der Gruppe der Dozierenden, welche ausschliesslich angehende Sekundarlehrpersonen unterrichtet, sind in der Deutschschweiz nur rund ein Fünftel aller Dozierenden weiblich (21\%). Einzig im Oman unterrichten mit einem Anteil von 5\% weniger Frauen als in der Deutschschweiz.

Betrachtet man die Länder über alle Gruppen hinweg, finden sich in Russland und den Philippinen ein besonders grosser Anteil an weiblichen Dozierenden; in der Deutschschweiz, in Taiwan und im Oman ist der Anteil an männlichen Dozierenden auffallend hoch. 
Abbildung 5.1: Geschlechtsspezifische Verteilung der Dozierenden im internationalen Vergleich dargestellt nach den Zielstufen

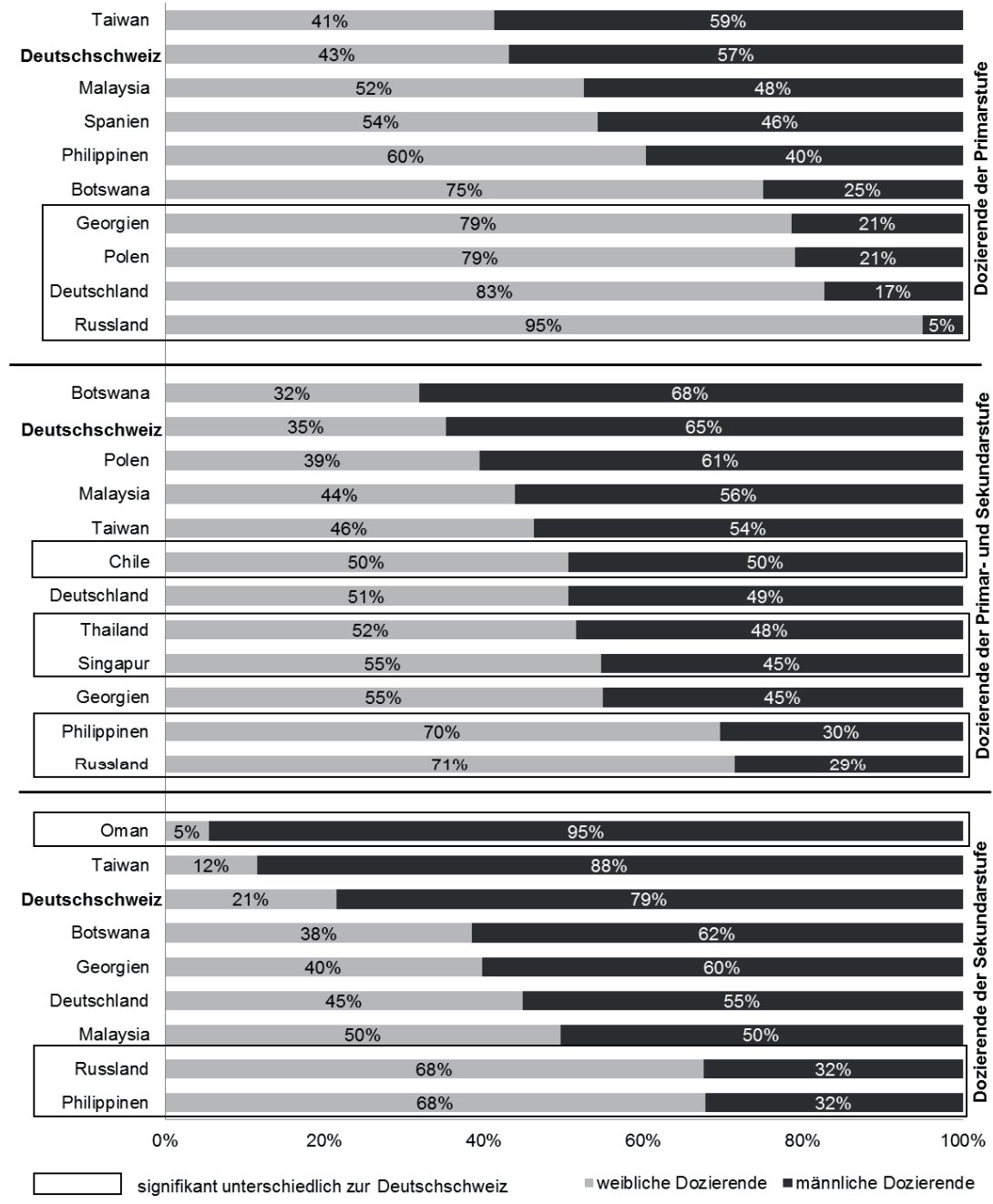

Anmerkungen: Die Länder wurden in Bezug auf die Geschlechterverteilung innerhalb der Zielstufen sortiert. Die Summe der Prozentwerte ergibt aufgrund von Rundungsfehlern nicht überall $100 \%$.

In Chile, Deutschland, in der Deutschschweiz und Malaysia ist die kombinierte Rücklaufquote kleiner als $60 \%$. In Polen ist die kombinierte Rücklaufquote kleiner als $75 \%$. Teilstichproben (Land/Zielstufe) $\mathrm{n}<10$ werden in der Darstellung nicht abgebildet. 
Bei den Deutschschweizer Dozierenden sticht besonders ein Resultat ins Auge. Der Anteil der männlichen Dozierenden ist bei der Erziehungswissenschaft mit der Zielstufe Sekundarstufe mit 86\% signifikant höher als in den anderen Teilgruppen (siehe Abbildung 5.2). Dieses Resultat korrespondiert mit dem Phänomen bei den Lehrpersonen der Volksschule: je höher die Zielstufe, desto grösser fällt der Männeranteil aus (Annen et al., 2010). Bei der Gruppe der Mathematikdozierenden kann jedoch höchstens eine Tendenz eines höheren männlichen Anteils in der Sekundarstufe im Vergleich zur Primarstufe festgestellt werden.

Abbildung 5.2: Geschlechtsspezifische Verteilung der Dozierenden der Deutschschweiz dargestellt nach den Fachgruppen und den Zielstufen

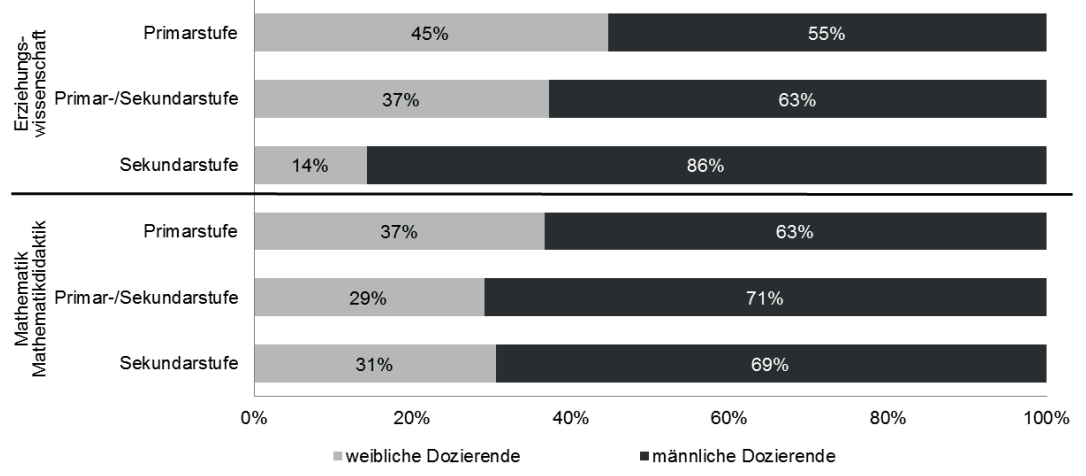

Criblez (2001) fand in der 1995/96 durchgeführten Studie 39.4\% weibliche Dozierende bei den Ausbildungsgängen für Lehrkräfte des Kindergartens und der Primarschule, was ungefähr den in TEDS-M erhobenen Zahlen entspricht. Bei den Dozierenden für die Lehrkräfte der Sekundarstufe I und II lag der Anteil 1995/96 bei 14.2\%. Dieser Anteil scheint etwas grösser geworden zu sein. Es gilt jedoch zu beachten, dass die Stichproben nicht direkt miteinander verglichen werden können, da einerseits die Zahl bei Criblez auch die Dozierenden mit Zielstufe Sekundarstufe II umfasst und andererseits in TEDS-M die Unterteilung zwischen Dozierenden der Primar- und Sekundarstufe nicht vollständig möglich ist.

Die „Feminisierung“, wie sie bei den Volksschullehrpersonen derweil in der Öffentlichkeit sorgenvoll diskutiert wird (Guggenbühl, 2001; Ruchti \& Meier, 2009), kann anhand der Daten bei den Dozierenden (noch) nicht beobachtet werden. Um eine gesicherte Aussage machen zu können, müsste man die Entwicklung der Daten jedoch über längere Zeiträume betrachten (Eckert, 2006). 


\subsubsection{Akademischer Hintergrund der Dozierenden}

\section{Abbildung 5.3: Höchster akademischer Abschluss der Dozierenden im internationalen Vergleich dargestellt nach Zielstufen (Angaben in Prozent)}

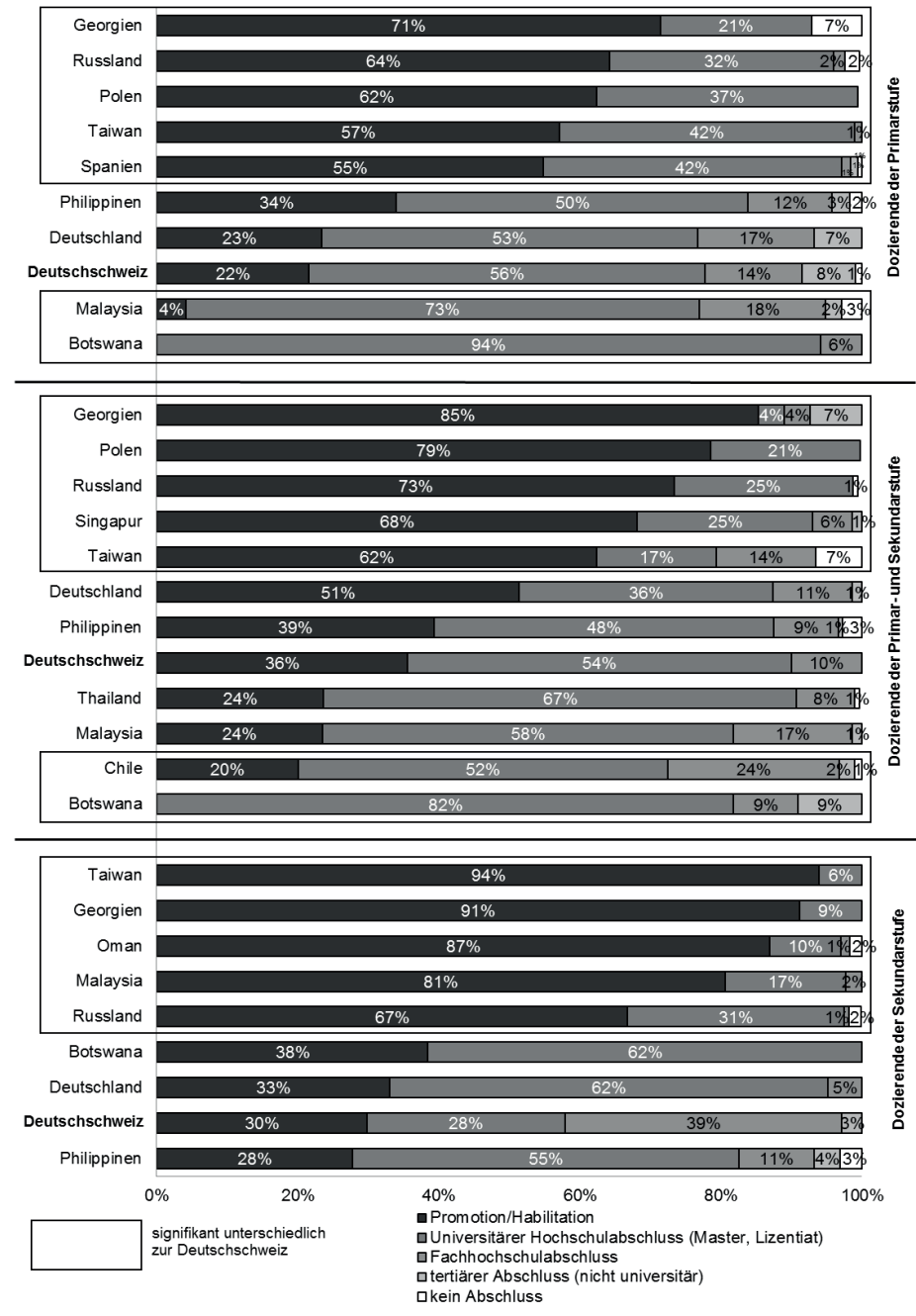

Anmerkungen: Die Länder wurden in Bezug auf den Anteil von Promotion/Habilitationen innerhalb der Zielstufen sortiert. Die Summe der Prozentwerte ergibt aufgrund von Rundungsfehlern nicht überall $100 \%$. In Chile, Deutschland, in der Deutschschweiz und Malaysia ist die kombinierte Rücklaufquote kleiner als $60 \%$. In Polen ist die kombinierte Rücklaufquote kleiner als $75 \%$. Teilstichproben (Land/Zielstufe) $n<10$ werden in der Darstellung nicht abgebildet. 
Ein wesentliches Merkmal der Charakterisierung der Dozierenden in der Lehrerausbildung ist deren akademische Qualifikation. Die Dozierenden haben in den Fachrichtungen Mathematik/Mathematikdidaktik, Erziehungswissenschaft, einem der Mathematik nahestehenden Fachbereich oder in einem anderen Fachbereich jeweils angegeben, welchen höchsten akademischen Abschluss sie erreicht haben. Um die Qualifikationen über die Länder hinweg vergleichen zu können, hat sich TEDS-M an den Stufen der Internationalen Standardklassifikation in Erziehung (ISCED ${ }^{6}$ ) orientiert. Dementsprechend standen den Dozierenden folgende Antwortmöglichkeiten zur Auswahl: kein Abschluss; nichtuniversitärer, tertiärer Abschluss wie beispielsweise an Fachschulen, an Lehrerseminarien, in der Reallehrerausbildung (ISCED-Level 5B); Fachhochschulabschluss wie z.B. Bachelor, Sekundarlehrerausbildung universitär oder an einer Pädagogischen Hochschule (erster Abschluss ISCEDLevel 5A); universitärer Hochschulabschluss wie etwa Master, Lizentiat (zweiter Abschluss ISCED-Level 5A); Promotion oder Habilitation (ISCEDLevel 6).

In Abbildung 5.3 werden die höchsten akademischen Abschlüsse der Dozierenden über alle Fachgebiete hinweg im Ländervergleich, aufgeteilt nach den Zielstufen der unterrichteten Studierenden, dargestellt. Der Anteil an Dozierenden mit einer Habilitation oder einem Doktorat variiert zwischen 0\% (Botswana) und 94\% (Taiwan, Zielstufe Sekundarstufe). Grundsätzlich kann eine Tendenz zu einem grösseren Anteil von Abschlüssen auf ISCD-Level 6 (Doktorat/Habilitation) auf höherer Zielstufe Sekundarstufe festgestellt werden. In Malaysia sind die Unterschiede zwischen den Dozierenden für die Primarstufe (Anteil Dozierende mit Doktorat/Habilitation: 4\%) und für die Sekundarstufe (Anteil Dozierende mit Doktorat/Habilitation: 81\%) besonders ausgeprägt, aber auch in Botswana, Taiwan oder Georgien sind die Unterschiede beträchtlich. Dies deutet darauf hin, dass in diesen Ländern von den Dozierenden der Sekundarstufe eine höhere akademische Qualifikation verlangt wird.

Die Deutschschweiz liegt bezüglich der Habilitations- und Promotionsabschlüsse jeweils im unteren Drittel. Georgien, Russland, Taiwan, Spanien, Polen und der Oman weisen signifikant höhere Anteile an Dozierenden bei den höchsten Abschlüssen auf. Eine geringere Quote bei den Abschlüssen als die Deutschschweiz zeigen bei der Zielstufe Primarstufe Malaysia und Botswana und bei der gemischten Zielstufe Chile und Botswana. Auf der Zielstufe Sekundarstufe besitzt die Deutschschweiz (zusammen mit den Philippinen) die tiefste Rate an Habilitationen/Promotionen.

In allen Ländern und über alle Zielstufen hinweg verfügen mindestens $72 \%$ der Dozierenden mindestens über einen universitären Hochschulabschluss (also zumindest Lizentiat oder Master), was auf eine hohe Qualifikation der Dozierenden hinweist. Es existiert aber eine Ausnahme von besonderer Bri-

6 International Standard Classification of Education. 
sanz. Nur 58\% der Deutschschweizer Dozierenden, welche ausschliesslich Studierende für die Sekundarstufe ausbilden, haben wenigstens den Abschluss eines Lizentiats oder Masters. 39\% aller Dozierenden, welche ausschliesslich auf der Sekundarstufe unterrichten, besitzen höchstens einen Fachhochschulabschluss, 3\% sind gar nur im Besitz eines nichtuniversitären tertiären $\mathrm{Ab}$ schlusses. Somit ist die Gruppe der Deutschschweizer Dozierenden mit ausschliesslicher Zielstufe Sekundarstufe deutlich weniger akademisch qualifiziert als alle anderen Ländergruppen.

Abbildung 5.4: Höchste akademische Abschlüsse der Dozierenden der Deutschschweiz dargestellt nach den Fachgruppen und den Zielstufen

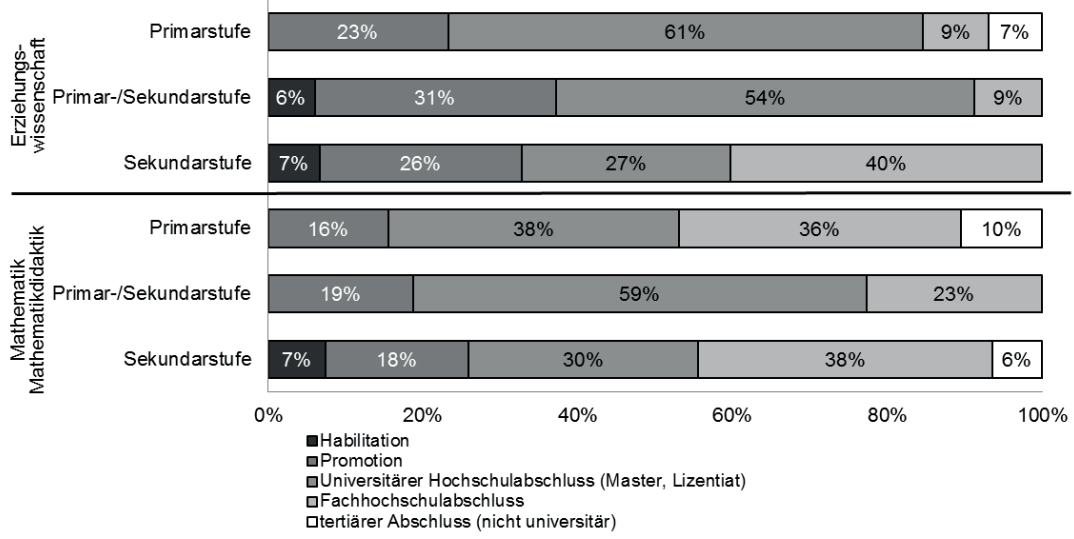

In der Deutschschweiz wurden die Daten so erhoben, dass eine Unterscheidung zwischen Promotion und Habilitation möglich ist. In der Stichprobe besitzen nur Dozierende, die auf beiden Stufen oder ausschliesslich auf der Sekundarstufe tätig sind, eine Habilitation. In der Stichprobe der Dozierenden, welche ausschliesslich Primarstufenlehrpersonen ausbilden, findet sich keine Person mit einer Habilitation. Der Anteil an Promotionen variiert zwischen 16 und 31\%. Diese Differenzen stellen keine signifikanten Unterschiede zwischen den Gruppen dar. Besonders fällt bei den Dozierenden der Sekundarstufe der grosse Anteil an Dozierenden mit Fachhochschulabschluss auf (Erziehungswissenschaft 40\%, Mathematik/Mathematikdidaktik 38\%). Die Gruppe der Dozierenden, welche nur Studierende der Sekundarstufe unterrichtet, scheint in Bezug auf ihre höchsten akademischen Abschlüsse heterogener als die anderen Dozierendengruppen zu sein.

Cirblez (2001) stellte in den Daten von 1995/96 fest, dass die Ausbildnerinnen bezüglich der formalen Qualifikation den Ausbildnern unterlegen sind. Dieses 
Resultat kann mit den hier vorliegenden Daten von TEDS-M nicht repliziert werden (siehe Abbildung 5.5). Der Anteil an Dozentinnen mit einem universitären Hochschulabschluss ist gering höher als derjenige der Dozenten, was eine Veränderung gegenüber den Ergebnissen von Criblez aufzeigen würde. Möglicherweise sind die weiblichen Dozierenden in den Studiengängen für angehende Primar- oder Sekundarlehrpersonen 2008 besser qualifiziert, als dies 1995/96 noch der Fall war. ${ }^{7}$

Abbildung 5.5: Höchste akademische Abschlüsse der Dozierenden der Deutschschweiz dargestellt nach dem Geschlecht

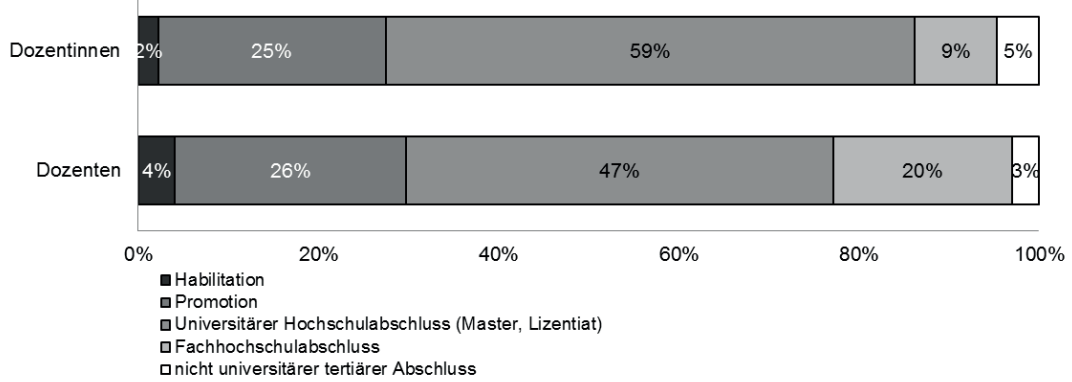

Im Reglement über die Anerkennung von Hochschuldiplomen für Lehrkräfte der Vorschulstufe und der Primarstufe vom 10. Juni 1999 der Schweizerischen Konferenz der kantonalen Erziehungsdirektoren (EDK) steht in Artikel 6, dass die Dozentinnen und Dozenten u.a. über einen Hochschulabschluss im zu unterrichtenden Fachgebiet verfügen sollten, mit der Ergänzung: „Vom Hochschulabschluss kann im Einzelfall insbesondere in den Bereichen Stufen- und Fachdidaktik abgewichen werden, sofern die fachliche Eignung auf andere Art nachgewiesen wird“ (EDK, 1999b, S.7). Bei den Dozierenden, die ausschliesslich Studierende der Primarstufe unterrichten, erfüllen im Fachbereich Erziehungswissenschaft 84\% die Anforderung eines Hochschulabschlusses. Bei den Dozierenden für Mathematik und Mathematikdidaktik weisen nur 53\% einen Hochschulabschluss auf, was einem signifikanten Unterschied entspricht. Ob die restlichen $47 \%$ die Zusatzklausel bezüglich einer fachlichen Eignung anderer Art erfüllen, kann anhand der hier vorliegenden Daten nicht beurteilt werden.

Im Reglement über die Anerkennung von Hochschuldiplomen für Lehrkräfte der Sekundarstufe I vom 26. August 1999 der Schweizerischen Konferenz der kantonalen Erziehungsdirektoren (EDK) steht in Artikel 7, dass die Dozierenden u.a. über einen Hochschulabschluss im zu unterrichtenden Fach-

7 Die Stichprobenzusammensetzung in der Studie von Criblez (2001) umfasst Studiengänge, die bei TEDS-M nicht miteinbezogen wurden. Deshalb sind die Resultate nur unter grösstem Vorbehalt miteinander vergleichbar. 
gebiet verfügen sollen. Für Fachdidaktikerinnen und -didaktiker wird darüber hinaus entweder eine Promotion in Fachdidaktik oder ein Lehrdiplom und Unterrichtserfahrung eingefordert (EDK, 1999a). Wird die Gruppe der Dozierenden betrachtet, welche ausschliesslich auf der Zielstufe Sekundarstufe I unterrichtet, erfüllt ein relativ grosser Anteil (Erziehungswissenschaft 40\%, Mathematik/Mathematikdidaktik 44\%) die Forderung nach einem Hochschulabschluss nicht. Einen signifikant höheren Anteil an Hochschulabschlüssen weisen die Dozierenden auf, die Studierende für beide Stufen unterrichten (Erziehungswissenschaft 81\%, Mathematik/Mathematikdidaktik 77\%).

Die im internationalen Vergleich gesehenen, relativ bescheidenen akademischen Qualifikationen der Deutschschweizer Dozierenden müssen auf dem Hintergrund der erst kürzlich erfolgten Tertiarisierung der Lehrerausbildung betrachtet werden. Die ehemaligen Lehrerseminarien stellten geringere Qualifikationsanforderungen an ihre Dozierenden als die heutigen Pädagogischen Hochschulen. Entsprechend den Regelungen der Kantone wurde eine grosse Anzahl ehemaliger Seminarlehrerinnen und Seminarlehrer von den Pädagogischen Hochschulen übernommen (Annen et al., 2010; EDK, 2008). Dieser Umstand erklärt mindestens einen Teil der tieferen Qualifikationen.

Auffällig bleibt jedoch die tiefe Qualifikation der Dozierenden der Sekundarstufe im nationalen wie auch im internationalen Vergleich, wobei relativierend erwähnt werden muss, dass die Fallzahlen bei den Sekundarstufendozierenden klein sind. ${ }^{8}$ Bereits vor der Tertiarisierung der Lehrerbildung waren mehrere Sekundarlehrerausbildungen an Universitäten angesiedelt (Criblez, 2001). Es kann sein, dass Dozierende der früheren universitären Sekundarlehrerausbildung nicht an Pädagogische Hochschulen gewechselt haben und sich somit eine Personallücke gebildet hat. Eine mögliche weitere Erklärung lässt sich finden, wenn man die Lehrbefähigungen der Dozierenden mitberücksichtigt (siehe Kapitel 5.4.3). Die Dozierenden mit der Zielstufe Sekundarstufe verfügen über einen hohen Anteil an Lehrdiplomen für die Sekundarstufe I oder II, Dozierende mit der Zielstufe Primarstufe besitzen einen hohen Anteil an Lehrdiplomen für die Primarstufe. Im Unterschied zum früheren Primarlehrerdiplom sind die Lehrdiplome der Sekundarstufe I und II im tertiären Bereich erworben. Möglicherweise ist es für Personen mit einem Primarlehrerdiplom attraktiver, sich über ein Universitätsstudium weiterzubilden als für Personen mit einem Sekundarlehrdiplom. Es könnte für ehemalige Primarlehrpersonen wichtiger sein, sich universitär auszubilden, um in der Lehrerausbildung tätig zu sein, als es dies für Sekundarlehrpersonen ist. Eine weitere Erklärung für die tiefere Qualifikation der Dozierenden könnte schlicht ein Rekrutierungsproblem sein. In der Schweiz sind erst zaghafte Versuche zur Einrichtung von einzelnen Fachdidaktik-Lehrstühlen zu erkennen, was zur

8 Je sieben Sekundarstufen-Dozierende bei den Erziehungswissenschaft und der Mathematik haben höchstens einen Fachhochschulabschluss. 
Folge hat, dass Dozierende mit einer Promotion in Fachdidaktik bis anhin nur im deutschsprachigen Ausland gefunden werden konnten.

In Anschlussbericht zum Masterplan formuliert die EDK (EDK, 2008), dass in den nächsten Jahren Anstrengungen zur Weiter- und Nachqualifikation der Dozierenden anstehen. Ebenfalls soll bei Neuanstellungen darauf geachtet werden, dass die Qualifikationsanforderungen so eingehalten werden können, dass „die Dozierenden der Pädagogischen Hochschulen (...) auf hohem Niveau wissenschaftlich, fachlich, und fachdidaktisch qualifiziert" (EDK, 2008, S. 6) sind.

\subsubsection{Schulische Lehrbefähigung der Dozierenden}

Die schulische Lehrbefähigung auf einer der Zielstufen zeigt auf, ob die Dozierenden selbst einmal den Beruf der Lehrperson erlernt haben und gilt als ein Aspekt für die Bemessung der praktischen Qualifikation der Dozierenden. Da es in einigen Ländern möglich ist, dass das Lehrdiplom seine Gültigkeit verliert, wurde in TEDS-M zusätzlich der Status der Lehrbefähigung dreigegliedert mit a) im Besitz eines aktuellen Lehrdiploms, b) war im Besitz eines Lehrdiploms und c) war nie im Besitz eines Lehrdiploms.

Der Anteil der Dozierenden, welche aktuell ein Lehrdiplom besitzen, variiert international zwischen 22\% (Taiwan, Zielstufe Sekundarstufe) und 100\% (Georgien, Zielstufe Primarstufe) (siehe Abbildung 5.6). Bei der Zielstufe Primarstufe weisen die Dozierenden in sieben von zehn Ländern einen Lehrdiplomanteil von über 75\% auf, wozu auch die Deutschschweiz mit einem Anteil von $89 \%$ gehört. Polen und Taiwan unterscheiden sich von der Deutschschweiz mit tieferen Werten, Georgien mit höheren Werten zudem signifikant. Bei den Dozierenden mit beiden Zielstufen besitzen 90\% der Deutschschweizer ein Lehrdiplom. Zusammen mit Georgien, Russland, Chile, Malaysia und Botswana liegen die Deutschschweizer damit an der Spitze dieser Auflistung. Bei der Sekundarstufe weisen gar 97\% der Deutschschweizer Dozierenden ein Lehrdiplom auf, womit sie sich deutlich von Russland, den Philippinen, dem Oman, Malaysia und Taiwan abheben. 
Abbildung 5.6: Dozierende mit Lehrdiplom für die Primar- und Sekundarstufe im internationalen Vergleich aufgeteilt nach Zielstufen

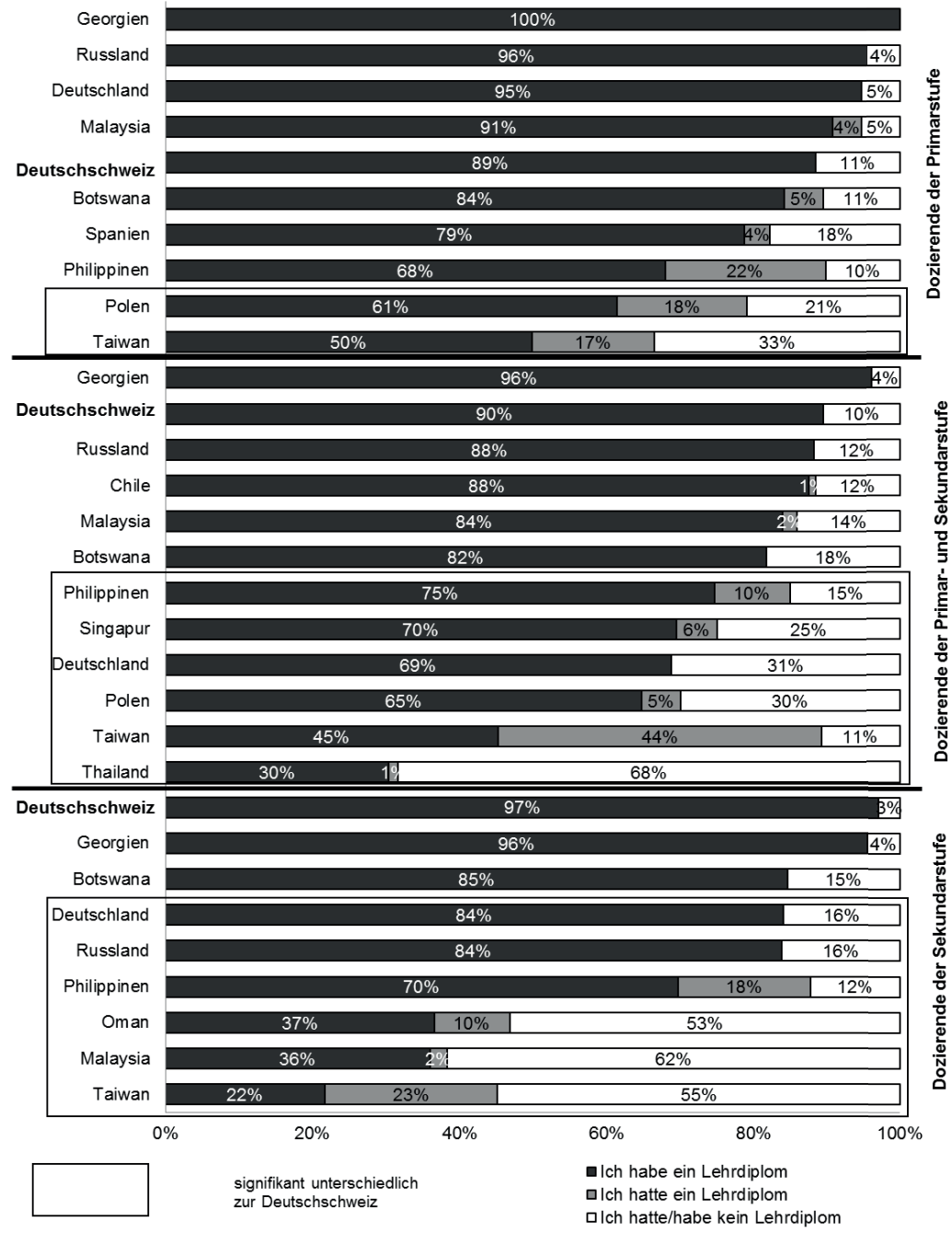

Anmerkungen: Die Länder wurden in Bezug auf den Anteil Dozierender mit Lehrdiplom innerhalb der Zielstufen sortiert. Die Summe der Prozentwerte ergibt aufgrund von Rundungsfehlern nicht überall $100 \%$. In Chile, Deutschland, in der Deutschschweiz und in Malaysia ist die kombinierte Rücklaufquote kleiner als 60\%. In Polen ist die kombinierte Rücklaufquote kleiner als $75 \%$. Teilstichproben (Land/Zielstufe) $\mathrm{n}<10$ werden in der Darstellung nicht abgebildet. 
Abbildung 5.7: Dozierende der Deutschschweiz mit oder ohne Lehrdiplom für die Primar-, Sekundarstufe und für das Gymnasium dargestellt nach den Fachgruppen und den Zielstufen

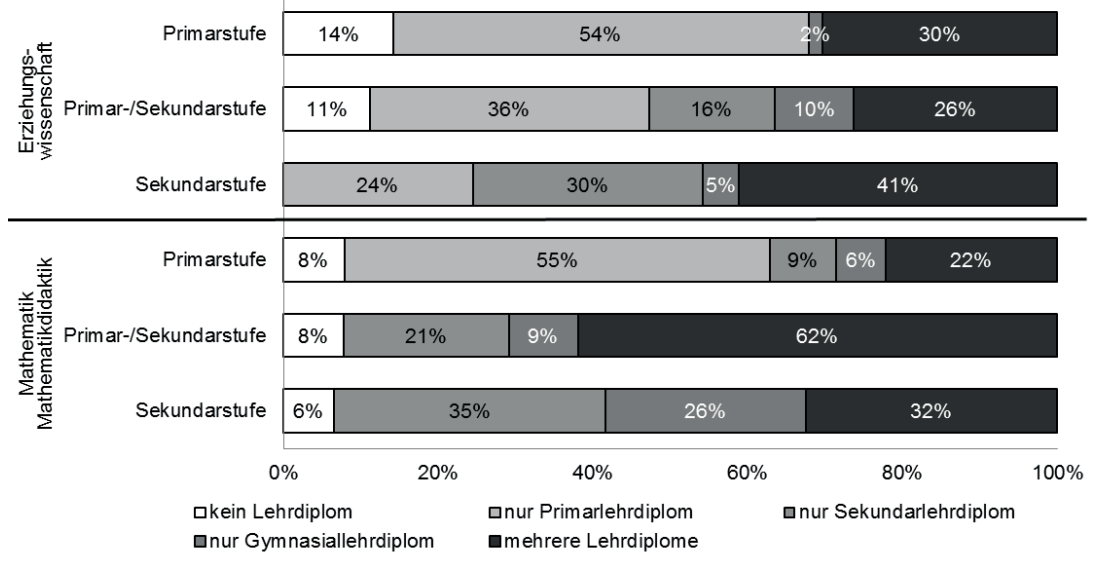

Allgemein kann festgestellt werden, dass in der Deutschschweiz der Anteil an Dozentinnen und Dozenten mit Lehrdiplom auf der Sekundarstufe im Vergleich zur Primarstufe höher liegt. Dieser Befund verhält sich konträr zu den meisten anderen Ländern. Die Anteile der Dozierenden mit Lehrdiplom fallen auf der Primarstufe teilweise deutlich höher als auf der Sekundarstufe (bspw. Malaysia, Taiwan) aus. Es muss jedoch angemerkt werden, dass bei den Dozierenden der Primarstufe der Anteil ,kein Lehrdiplom“ in Wirklichkeit wahrscheinlich kleiner ist, da davon ausgegangen werden kann, dass vor allem in den Studiengängen Kindergarten-Unterstufe eine unbekannte Zahl von Dozierenden ein Kindergartendiplom besitzt. Die Angaben über den Besitz eines Kindergartenlehrdiploms wurden leider nicht erhoben.

In der Deutschschweiz werden die Dozierenden zusätzlich nach der Art ihrer Lehrdiplome befragt (Primarlehrdiplom, Sekundarlehrdiplom oder Gymnasiallehrdiplom; siehe Abbildung 5.7 $)$. Zwischen 22 bis $62 \%$ der Dozierenden verfügen über mehrere Lehrdiplome. Dozierende, welche ausschliesslich das Primarlehrerdiplom erworben haben, unterrichten im Fachbereich Mathematik nur auf der Primarstufe, bei der Erziehungswissenschaft in etwas höherem Umfang auf der Primarstufe, aber, im Unterschied zum Fachbereich Mathematik/Mathematikdidaktik, auch auf den anderen beiden Zielstufen.

Nicht nur ein Lehrdiplom, sondern das passende, also das der Zielstufe entsprechende Lehrdiplom ist bedeutungsvoll (siehe Abbildung 5.8). In der Deutschschweizer Stichprobe schwankt der Anteil von Dozierenden mit einem Lehrdiplom für die Zielstufe zwischen 62 und 83\%. Es existieren keine signi-

9 In der Studie wurde nicht nach dem Besitz von Kindergartenlehrdiplomen gefragt. 
fikanten Unterschiede zwischen den Gruppen. Bei den Dozierenden der Zielstufe Primarstufe wäre der Anteil von Lehrdiplomen für die Zielstufe wahrscheinlich noch grösser, wenn die Kindergartendiplome ebenfalls berücksichtigt worden wären.

In der Schweiz wird von Dozierenden, welche Lehrpersonen für die Vorschulstufe und die Primarstufe ausbilden, nebst den akademischen Qualifikationen in der Regel ein Lehrdiplom erwartet (EDK, 1999b). Von Dozierenden, die angehende Lehrpersonen auf die Sekundarstufe vorbereiten, ist ein Lehrdiplom nicht explizit Bedingung, allerdings wird von ihnen im Bereich der Fachdidaktik entweder eine Promotion oder ein Lehrdiplom gefordert (EDK, 1999a). Aufgrund der hier vorliegenden Daten kann nicht schlüssig beurteilt werden, ob die Regelung der EDK vollumfänglich erfüllt werden, da die Daten zu den Kindergartendiplomen fehlen. Jedoch kann festgestellt werden, dass ein sehr grosser Anteil der Dozierenden (total 89\%) mindestens ein Lehrdiplom besitzt. 30\% aller Dozierenden sind gar im Besitz von mehreren Lehrdiplomen.

Abbildung 5.8: Dozierende der Deutschschweiz mit Lehrdiplom für die Zielstufe dargestellt nach den Fachgruppen und den Zielstufen

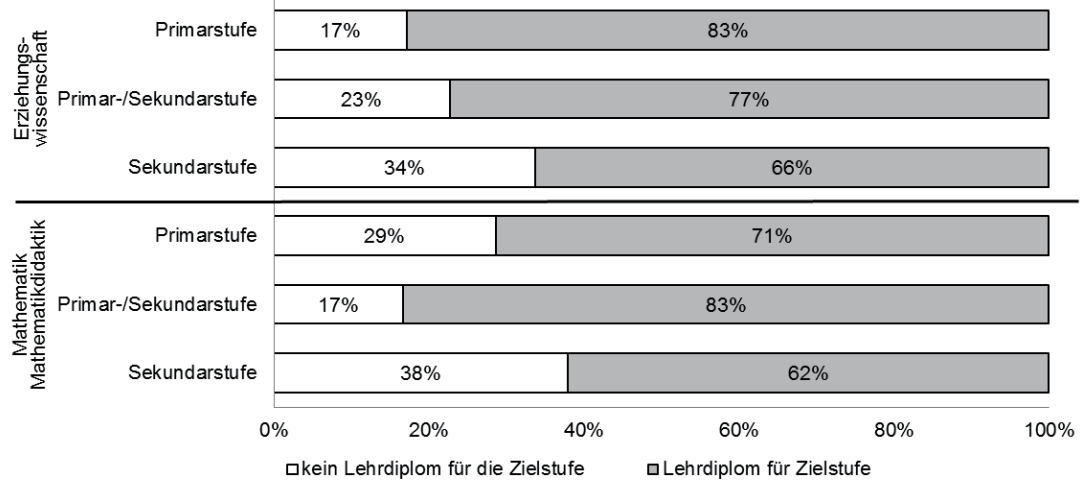

\subsubsection{Ausbildung der Dozierenden als Lehrerausbildende}

Verschiedene Forschungen zeigen, dass es im europäischen Raum kaum spezifische Ausbildungen für Lehrerausbildende gibt (Korthagen et al., 2005; Noel, 2006). Noel (2006) spricht gar vom ,secret life“ der englischen Lehrerausbildenden und meint damit, dass kaum bekannt ist, wie jemand überhaupt Lehrerausbildender wird. Auch in der Deutschschweiz existiert keine explizite Ausbildung für Lehrerausbildende. Es bestehen aber Angebote zur freiwilligen Weiterbildung wie beispielsweise ein Nachdiplomstudiengang für Dozierende 
an Pädagogischen Hochschulen (bspw. MAS of Advanced Studies in Teacher Education).

Im Zuge der Tertiarisierung der Lehrerausbildung wurden in der Schweiz durch die EDK (EDK, 1999a; EDK, 1999b) Bestimmungen zur formalen Qualifikation der Lehrerausbildenden erlassen. Diese fordern einen Hochschulabschluss im zu unterrichtenden Fach und allenfalls zusätzlich Lehrdiplome oder Promotionen (siehe Kapitel 5.4.3). Eine spezifische Ausbildung für Lehrerausbildende wird jedoch nicht verlangt.

Abbildung 5.9: Dozierende mit/ohne Ausbildung als Lehrerausbildende und ihre akademischen Abschlüsse

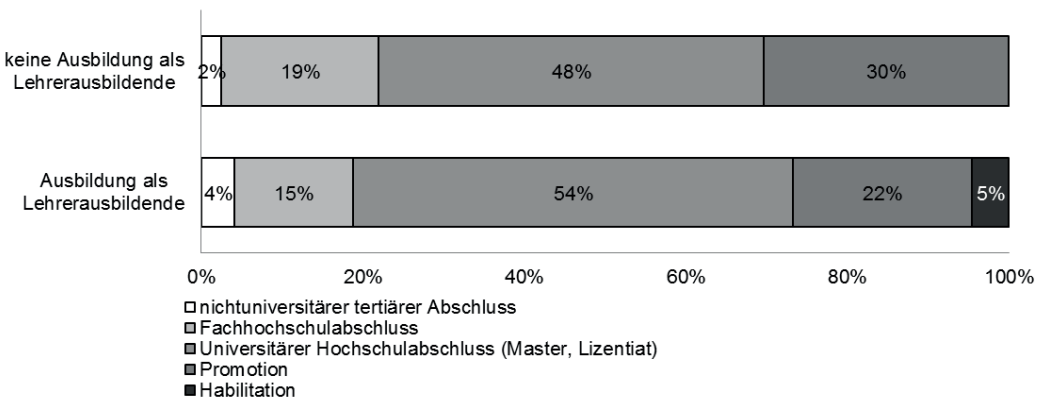

Vor der Reform in der Lehrerbildung zeichnete ,sich die Situation der LLB in der Schweiz [...] durch grosse Pluralität, ja sogar Beliebigkeit" (Criblez, 1994; zitiert nach Criblez, 2010) aus, was sich u.a. auch bei den Anforderungen an die Lehrerausbildenden widerspiegelte. Von Seminarlehrpersonen der Fachwissenschaften und Fachdidaktiken wurde häufig die Ausbildung als Gymnasiallehrerin oder -lehrer verlangt, während die Anforderungen für Dozierende im Bereich der Pädagogik, Psychologie und der allgemeinen Didaktik/ Methodik weit unklarer war. Bedeutende Akzente in der Ausbildung von Lehrerausbildenden in diesen Bereichen hatte Hans Aebli mit der Seminarlehrerausbildung LSEB (Lehrer und Sachverständiger für Erziehungs- und Bildungsfragen) an der Universität Bern gesetzt (Criblez, 2001).

In TEDS-M wurden die Dozierenden zusätzlich befragt, ,inwiefern sie für die Ausbildung angehender Lehrpersonen ausgebildet wurden“. In der Deutschschweiz geben 75\% aller Dozierenden an, dass sie als Lehrerausbildende ausgebildet wurden, was dem internationalen Mittel entspricht. Zwischen den sechs Teilgruppen der Deutschschweizer Dozierenden sind keine Unterschiede erkennbar. Da es in der Deutschschweiz keine eigentliche Ausbildung für Lehrerausbildende gibt, soll der Frage nachgegangen werden, wann sich Dozierende als ,,ausgebildet“ bezeichnen. Die akademische Qualifikation hat auf den selbst berichteten Ausbildungsstatus der Dozierenden erstaunlicherweise keinen Einfluss (siehe Abbildung 5.9). Analysiert man das 
Antwortverhalten bezüglich des Besitzes eines Lehrdiploms, kann eine Tendenz festgestellt werden, dass Dozierende, die angeben, sie hätten keine Ausbildung als Lehrerausbildende, weniger häufig über ein Lehrdiplom verfügen.

Das Antwortverhalten der Deutschschweizer Dozierenden lässt demnach verschiedene Interpretationen zu. Da die Fragestellung der international vorgegebenen Form nicht auf die Deutschschweizer Verhältnisse passte, wurde für die Befragten nicht ersichtlich, ob hier nach einer speziellen Ausbildung für Lehrerausbildende, nach dem Erfüllen der formalen Qualifikationen oder gar nach dem eigenen Gefühl der Qualifizierung gefragt wird. Die statistische Auswertung zeigt kein eindeutiges Bild auf und das Antwortverhalten der Dozierenden kann zu wenig mit den Gegebenheiten in der Deutschschweiz verbunden werden. Aufgrund dieser unklaren Situation wird in Bezug auf diese Frage von einem Vergleich im internationalen Kontext abgesehen.

\subsubsection{Lehrerfahrung in der Primar- und/oder Sekundarschule}

Die praktische Erfahrung als Lehrperson gilt als ein charakterisierendes Merkmal der Dozierenden der Lehrerausbildung. Die Kenntnis der Praxis soll gewährleisten, dass die Lehre den Bezug zur Realität ,Schule“ sicherstellt. Dass jedoch die Gleichung - eine gute Lehrperson gleich eine gute Lehrperson in der Lehrerausbildung - nicht gelten darf, wird in der Literatur bekräftigt (u.a. Korthagen et al., 2005; Zeichner, 2005). Die Art und Weise, wie Dozierende unterrichten, und diese ist sicherlich von den Erfahrungen als Lehrperson auf der Primar- und Sekundarstufe geprägt, kann die angehenden Lehrpersonen stark beeinflussen. So sagt Blume: ,Teachers teach as they are taught, and not as they are taught to teach" (1971) oder, wie es Russell kurz und knapp zusammenfasst: „How I teach IS the message“ (1997, S. 32).

In TEDS-M wurden die Dozierenden gebeten, ihre Lehrerfahrung in Anzahl der Berufsjahre auf der Primar- und der Sekundarstufe zu nennen. Für die Auswertung werden die Anzahl der Jahre zusammen gezählt und vier Kategorien zugeordnet (keine Lehrerfahrung, 1-5 Jahre Lehrerfahrung, 6-10 Jahre Lehrerfahrung, mehr als 10 Jahre Lehrerfahrung). Diese Form der Darstellung erlaubt die Ermittlung der Anteile an unerfahrenen, wenig erfahrenen, erfahrenen und sehr erfahrenen Dozierenden in Bezug auf die Unterrichtspraxis in der Schule.

Im internationalen Vergleich fällt auf, dass auf der Primarstufe ein grosser Anteil der Dozierenden Lehrerfahrung oder gar langjährige Lehrerfahrungen besitzt (siehe Abbildung 5.10), auf der Zielstufe Sekundarstufe aber ein beträchtlicher Anteil an Dozierenden über keine Lehrerfahrung verfügt. 
Abbildung 5.10: Lehrerfahrung auf der Primar- und Sekundarstufe der

Dozierenden im internationalen Vergleich dargestellt nach den Zielstufen

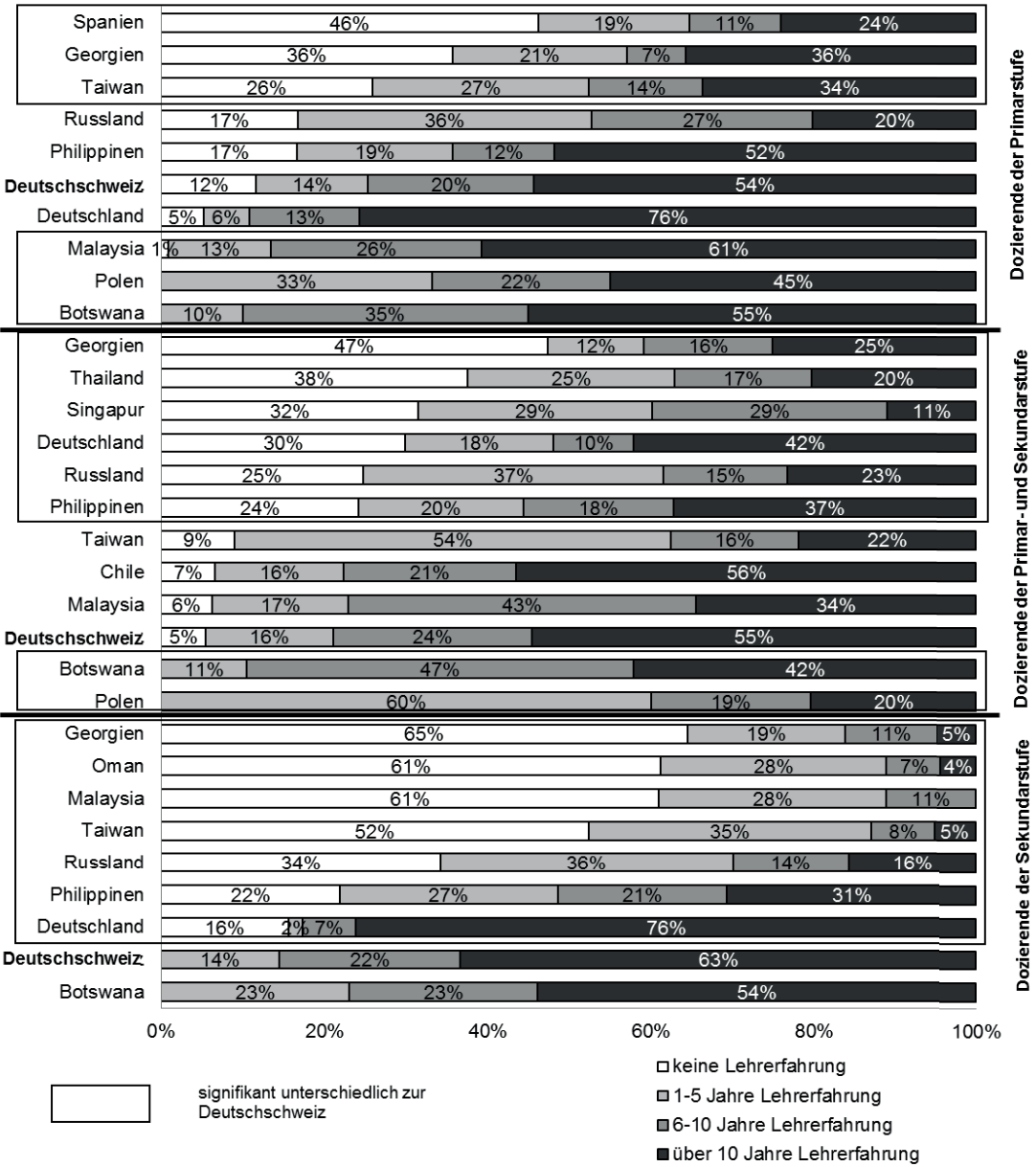

Anmerkungen: Die Länder wurden in Bezug auf "keine Lehrerfahrung“ innerhalb der Zielstufen sortiert. Die Summe der Prozentwerte ergibt aufgrund von Rundungsfehlern nicht überall $100 \%$.

In Chile, Deutschland, in der Deutschschweiz und in Malaysia ist die kombinierte

Rücklaufquote kleiner als 60\%. In Polen ist die kombinierte Rücklaufquote kleiner als $75 \%$. Teilstichproben (Land/Zielstufe) $\mathrm{n}<10$ werden in der Darstellung nicht abgebildet. 
Ordnet man die Antworten der Länder infolge der Aussage „,keine Lehrerfahrungen", liegt die Deutschschweiz bei der Ausbildung für die Primarstufe mit $12 \%$ im Mittelfeld. Deutlich mehr Dozierende ohne Lehrerfahrungen haben Spanien und Taiwan. In Malaysia, Polen und Botswana finden sich hingegen keine Dozierende ohne Lehrerfahrung. Auf der Zielstufe Sekundarstufe gibt es in der Deutschschweiz keine Lehrerausbildende ohne Lehrerfahrung, was im internationalen Vergleich (zusammen mit Botswana) eine Besonderheit darstellt. Auch der Anteil an Dozierenden mit besonders viel Lehrerfahrung ist in der Deutschschweiz mit 63\% sehr hoch, was auch für Deutschland und Botswana zutrifft.

Abbildung 5.11: Lehrerfahrung der Dozierenden der Deutschschweiz auf der Primar- und/oder Sekundarstufe dargestellt nach den Fachgruppen und den Zielstufen

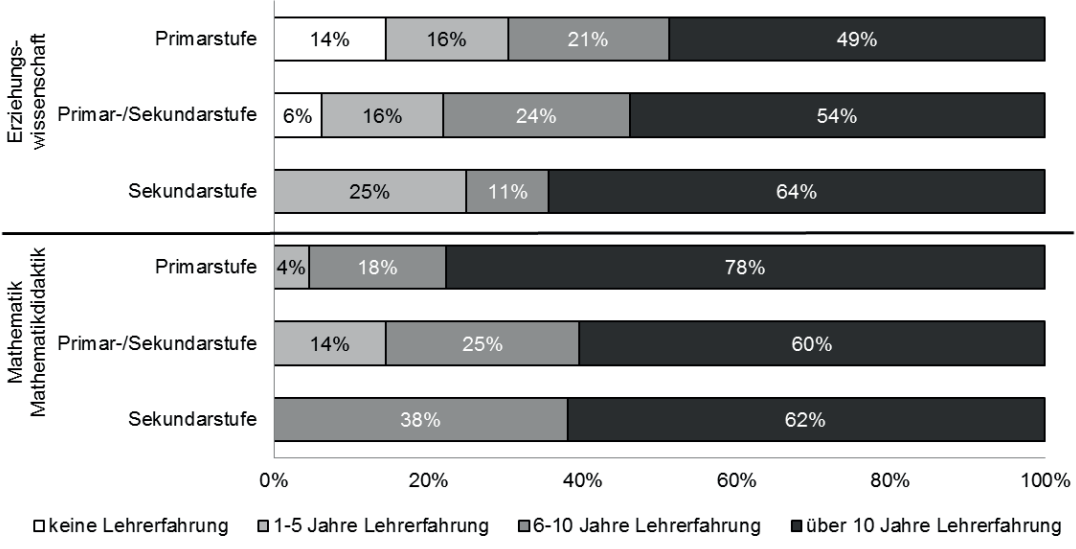

Betrachtet man die verschiedenen Gruppen der Dozierenden innerhalb der Deutschschweiz (siehe Abbildung 5.11), so verfügen nur einige Dozierende der Erziehungswissenschaft Primarstufe und beider Stufen über keine Lehrerfahrung. Allerdings ist es möglich, dass die Dozierenden Lehrerfahrungen auf der Kindergartenstufe gesammelt haben, was in TEDS-M leider nicht berücksichtigt wurde. Alle Dozierenden der Mathematik/Mathematikdidaktik haben mindestens ein Jahr Lehrerfahrung auf der Primar- oder Sekundarstufe, auf der Zielstufe Sekundarstufe mindestens 6 Jahre Lehrerfahrung. Gesamthaft gesehen verfügen die Dozierenden über einen grossen Erfahrungsschatz aus der Praxis. 
Abbildung 5.12: Lehrerfahrung der Deutschschweizer Dozierenden auf der Zielstufe der Studierenden dargestellt nach den Fachgruppen und den Zielstufen

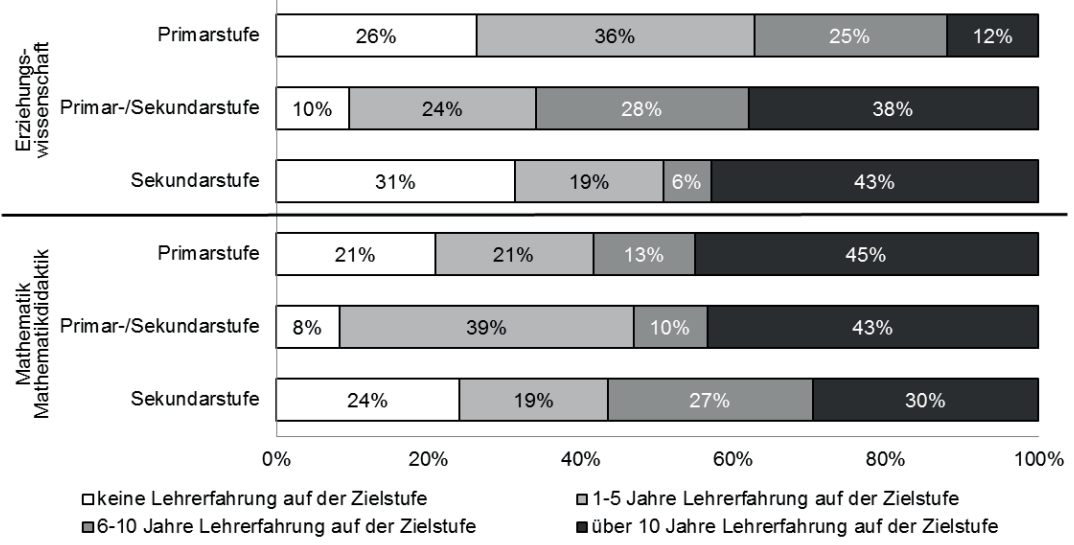

Betrachtet man nur die Lehrerfahrungen auf der entsprechenden Zielstufe (siehe Abbildung 5.12), ergibt sich ein anderes Bild. Von den Dozierenden, welche ausschliesslich auf der Sekundarstufe unterrichten, weisen 31\% (Erziehungswissenschaft) und 24\% (Mathematik/Mathematikdidaktik) keine Lehrerfahrungen auf der Zielstufe auf. In allen Teilgruppen haben Dozierende auch Erfahrungen auf anderen Schulstufen als der Zielstufe gesammelt.

\subsubsection{Forschungserfahrung der Dozierenden}

In der Schweiz zieht die Tertiarisierung der Lehrerausbildung einen Akademisierungsprozess mit sich, welcher sich folglich auch in einem Verwissenschaftlichungsprozess niederschlagen sollte. Die Pädagogischen Hochschulen sind, um ,hochschulwürdig“ zu sein, u.a. der Forschungs- und Wissenschaftsorientierung verpflichtet (Criblez, 2010). Die Forderung der Wissenschaftsorientierung war in der Diskussion um die neuen Pädagogischen Hochschulen nicht unumstritten. „Wissenschaftsorientierung und Persönlichkeitsbildung wurden als Gegensatz aufgebaut" (Criblez, 2010, S. 38) und man fragt(e) sich, ob ,die Kröte Wissenschaft" geschluckt werden müsse, ,um die Vorzüge von Professionalität und Ausbildung auf Hochschulniveau geniessen zu können“ (Herzog, 1999, S. 340f.). 
Abbildung 5.13: Forschungserfahrung der Dozierenden im internationalen Vergleich dargestellt nach den Zielstufen
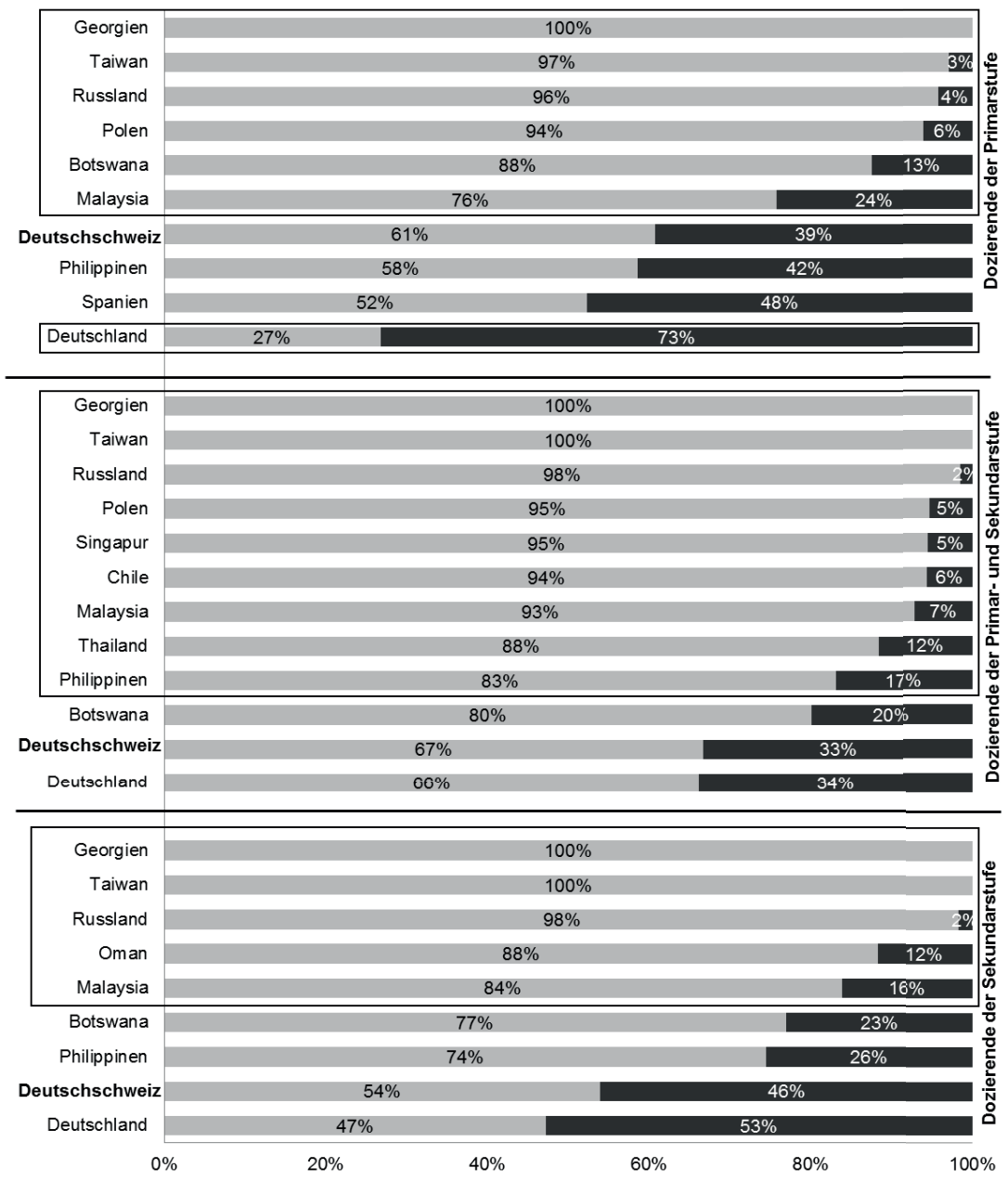

signifikant unterschiedlich zur Deutschschweiz $\quad$ Forschungserfahrung $\square$ keine Forschungserfahrung

Anmerkungen: Die Länder wurden nach dem Anteil der Forschungserfahrung innerhalb der Zielstufen sortiert. Die Summe der Prozentwerte ergibt aufgrund von Rundungsfehlern nicht überall $100 \%$.

In Chile, Deutschland, in der Deutschschweiz und in Malaysia ist die kombinierte

Rücklaufquote kleiner als $60 \%$. In Polen ist die kombinierte Rücklaufquote kleiner als $75 \%$.

Teilstichproben (Land/Zielstufe) $\mathrm{n}<10$ werden in der Darstellung nicht abgebildet. 
Um den durch die Hochschulwürde erhaltenen Auftrag der Forschung umsetzen zu können, müssen die Dozierenden entsprechende Qualifikationsanforderungen mitbringen. So schreibt die EDK im Beitrag für die Datenbank der Eurybase $^{10}$, dass ,genügend Fachdidaktik-Dozierende qualifiziert und die Forschung auf diesem Gebiet etabliert werden“" (EDK, 2007, S. 4) soll. Die Forschungserfahrung der Dozierenden ist ein Indiz dafür, inwiefern die Dozierenden zur Forschungsorientierung Pädagogischer Hochschulen beitragen können.

In TEDS-M wurden die Dozierenden gefragt, in welchen Fachrichtungen (Mathematik, Mathematikdidaktik, Bildungsforschung in anderen Gebieten als der Mathematikdidaktik) sie eigene Forschungserfahrungen ${ }^{11}$ gemacht haben. Aus den Antworten kann herausgelesen werden, wie gross der jeweilige Anteil der Dozierenden mit eigenen Forschungserfahrungen ist (siehe Abbildung 5.13). In Georgien und Taiwan haben alle oder fast alle Dozierenden eigene Forschungserfahrungen. Der geringste Anteil findet sich in Deutschland bei den Dozierenden, welche ausschliesslich auf der Zielstufe Primarstufe unterrichten.

Die Deutschschweiz liegt im internationalen Vergleich jeweils im unteren oder untersten Bereich. Nur 61\% der Dozierenden der Primarstufe, 67\% der Dozierenden beider Stufen und 54\% der Dozierenden der Sekundarstufe können auf eigene Forschungserfahrungen zurückgreifen.

Alle Länder, in denen die Studierenden bessere Leistungen in Mathematik und Mathematikdidaktik erreichen, haben signifikant mehr Dozierende mit Forschungserfahrungen als in der Deutschschweiz, wobei dies den Umkehrschluss nicht mit einschliesst.

\section{Abbildung 5.14: Forschungserfahrung der Deutschschweizer Dozierenden} dargestellt nach den Fachgruppen und den Zielstufen

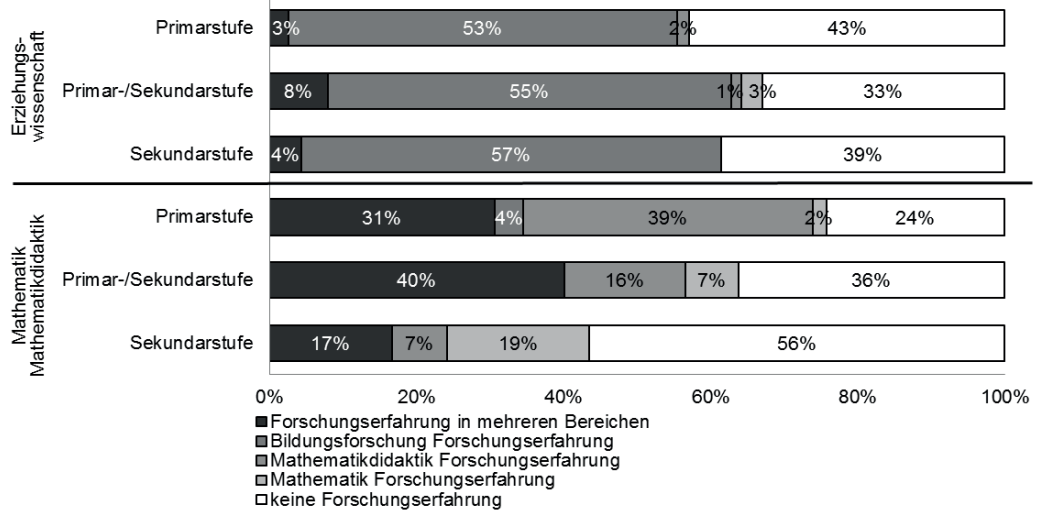

10 In der Datenbank Eurybase werden Informationen zu den Bildungssystemen der EU-Länder gesammelt.

11 Die Fragestellung schliesst Forschungserfahrungen im Rahmen einer Qualifikationsarbeit wie Lizentiat oder Masterarbeit nicht aus. 
Der Anteil an Dozierenden ohne Forschungserfahrung unterscheidet sich in der Deutschschweiz zwischen den verschiedenen Teilgruppen nicht signifikant, obwohl die Anteile zwischen 24 und 56\% variieren (siehe Abbildung 5.14). Die Erziehungswissenschaftsdozierenden verfügen, wie erwartet, vor allem über Erfahrungen im Bereich der Bildungsforschung, was einem Anteil von 53 bis 57\% der Dozierenden entspricht. Ein kleiner Anteil von 3 bis zu 8\% weist zusätzlich nicht nur Erfahrungen in der Bildungsforschung, sondern auch Erfahrungen in der Mathematik- oder Mathematikdidaktikforschung auf. Ein beträchtlicher Anteil (zwischen 17 und 40\%) der Dozierenden der Mathematik/Mathematikdidaktik verfügt in mindestens zwei Bereichen über Forschungserfahrungen. Forschung in mathematischen Themengebieten wird häufiger von Dozierenden der Sekundar- als der Primarstufe durchgeführt, Mathematikdidaktik wird hingegen tendenziell häufiger von Dozierenden der Primar- als der Sekundarstufe ergründet.

Wird die Zusammensetzungen der Teilgruppen nach Forschungserfahrungen betrachtet, finden sich bei den Dozierenden der Erziehungswissenschaft zwei Hauptgruppen: Dozierende mit Erfahrungen im Bereich der Bildungsforschung und Dozierende ohne Forschungserfahrung. Die Mathematikdozierenden weisen hingegen mehr Diversität in Bezug auf die Forschung auf: Es finden sich Dozierende ohne Forschungserfahrung, solche, die Forschung nur in der Mathematik betrieben und ein relativ grosser Anteil mit Forschungen gar in mehreren Gebieten.

Werden diese Ergebnisse mit dem Anliegen der Akademisierung und stärkeren Forschungsausrichtung der Lehrerbildung der Schweiz verglichen, muss es zu denken geben, dass über ein Drittel aller Dozierenden (37\%) über keine eigenen Forschungserfahrungen verfügt. Und dies, obwohl Dozierende möglicherweise auch einmalige Forschungserfahrung im Rahmen von Qualifikationsarbeiten wie Lizentiat oder Masterarbeit als eigene Forschungstätigkeit bewertet haben. Die tatsächliche Rate an Dozierenden mit eigener Forschungstätigkeit dürfte demnach wohl noch unter diesen 37\% liegen.

\subsubsection{Tätigkeitsprofil der Dozierenden}

Nebst dem Ausbildungs- und Erfahrungshintergrund untersucht TEDS-M auch das Tätigkeitsprofil der Dozierenden in ihrem beruflichen Alltag während der Lehrerausbildung von Lehrpersonen. Die Dozierenden wurden gebeten, anteilig zur Arbeitszeit an der Institution anzugeben, wie umfassend ihre Tätigkeiten in Lehre, Forschung, Verwaltung, Dienstleistung und sonstigen Arbeiten ausfallen. ${ }^{12}$

12 Bei der Frage wurde zusätzlich angemerkt, dass die Bereiche insgesamt $100 \%$ ergeben sollten. Jedoch hat nicht bei allen Dozierenden die Summe der Teilbereiche 100 Prozent ergeben. Um allfällige Verzerrungen zu vermeiden, wurden die Angaben der Dozierenden anteilig umgerechnet, so dass jeweils alle Angaben zusammen 100 Prozent ergaben. 
Mit durchschnittlich 57\% liegt die Haupttätigkeit der Deutschschweizer Dozierenden im Bereich der Lehre (siehe Abbildung 5.15). Die Dozierenden im Fachbereich Mathematik, die auf beiden Stufen lehren, investieren mehr Arbeitszeit für die Lehre als ihre Kolleginnen und Kollegen. Der Forschungsanteil schwankt je nach Teilgruppe zwischen 7 und 16\%, im Durchschnitt sind es knapp 11\%. Je 12\% der Arbeitszeit setzen die Dozierenden durchschnittlich für Verwaltungs- und Dienstleistungstätigkeiten ein. Weitere nicht näher bestimmbare Tätigkeiten beanspruchen $8 \%$ der Arbeitstätigkeit. ${ }^{13}$

Der Leistungsauftrag der Dozierenden ist dementsprechend in erster Linie auf die Lehre ausgerichtet und wird mit Arbeiten in der Verwaltung und der Dienstleistung ergänzt. Der relativ geringe Forschungsanteil an der Gesamtarbeitszeit hat möglicherweise damit zu tun, dass die meisten Lehrerbildungsinstitutionen der Schweiz Pädagogische Hochschulen und keine Universitäten sind und somit die Anstellung der Dozierende nicht auf die Forschung fokussiert ist, wie dies im universitären Rahmen üblich ist (Korthagen et al., 2005).

Abbildung 5.15: Tätigkeitsprofil der Dozierenden dargestellt nach den Fachgruppen und den Zielstufen

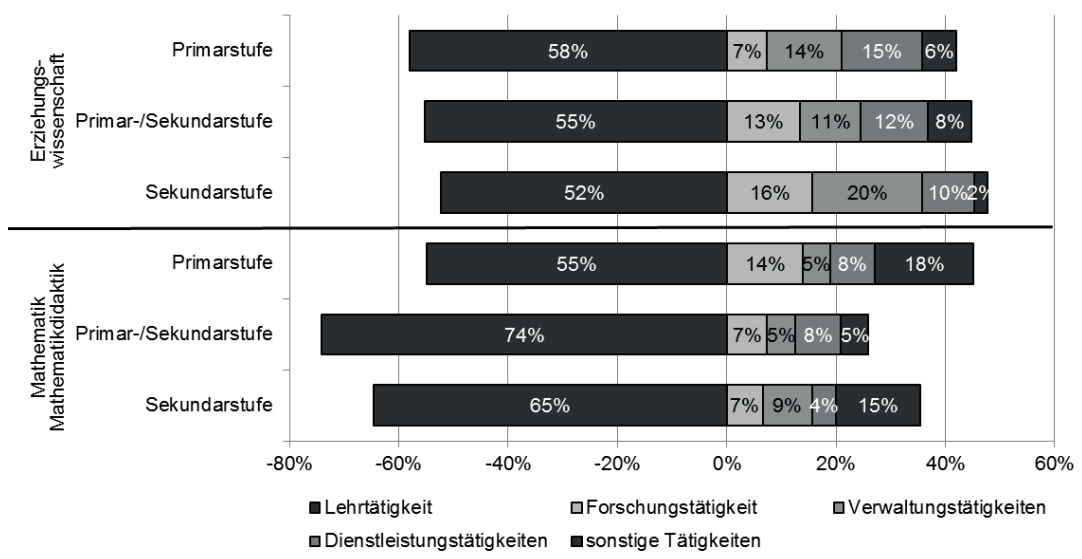

Um die Tätigkeiten präziser analysieren zu können, werden im Folgenden die Lehr- und Forschungstätigkeiten differenziert nach Fachgebiet und Zielstufe dargestellt. In Abbildung 5.16 wird der Umfang der Lehrtätigkeit an der Ge-

13 Entsprechend der Fragestellung ist nicht eruierbar, wie viel Arbeit tatsächlich in den verschiedenen Bereichen geleistet wird. Wenn beispielsweise Dozierende mit einem 20 Prozent-Pensum nur in der Lehre beschäftigt sind, fliesst dies als 100\% Lehrtätigkeit in die Berechnung ein. Deshalb darf hier explizit „,nur“ von den Tätigkeiten der Dozierenden gesprochen werden. Diese Zahlen spiegeln nicht die prozentual geleistete Arbeit in den Teilbereichen der Lehrerbildungsinstitutionen wider, da Personal, welches nicht in der Lehre tätig ist, nicht befragt wurde. 
samtarbeitszeit in vier Gruppen unterteilt: kleiner Anteil (Lehrtätigkeit unter 30\%), mittlerer Anteil (Lehrtätigkeit zwischen 31 und 50\%), grosser Anteil (Lehrtätigkeit zwischen 51 und 80\%) und sehr grosser Anteil an Lehrtätigkeit (über $80 \%$ ).

Abbildung 5.16: Anteil der Lehrtätigkeit an der Gesamtarbeitszeit der

Deutschschweizer Dozierenden dargestellt nach den Fachgruppen und den Zielstufen

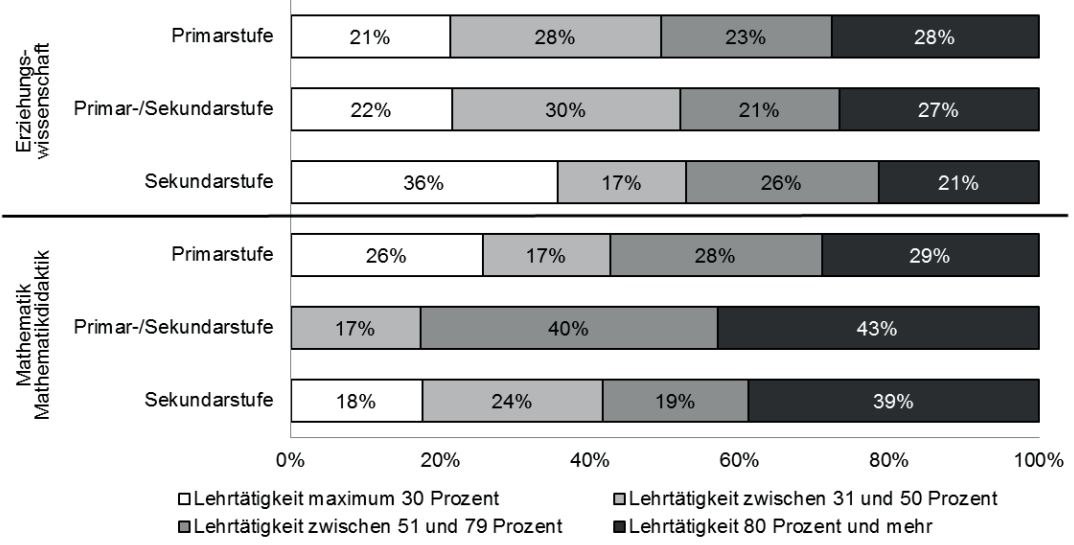

In der Gruppe der Mathematikdozierenden beider Stufen haben alle Dozierenden mindestens eine Lehrtätigkeit von 31\% inne. Tendenziell übt diese Gruppe häufiger eine Lehrtätigkeit im Umfang von 51 und mehr Prozent aus als die anderen Teilgruppen. In allen anderen Teilgruppen besitzt jeweils ungefähr ein Viertel der Dozierenden einen kleinen, mittleren, grossen oder sehr grossen Anteil in der Lehre. Es existiert also diesbezüglich kein typisches Dozierendenprofil, auch gibt es keine Unterschiede zwischen den Fachgebieten oder Zielstufen. Eine besondere Ausprägung findet sich lediglich bei den Mathematikdozierenden beider Stufen.

Die Mehrheit (53\%) der Deutschschweizer Dozierenden ist nicht in der Forschung tätig (siehe Abbildung 5.17). Ein Fünftel der Dozierenden verwendet bis $20 \%$ oder zwischen 20 bis 50\% seiner Arbeitszeit für Forschung. Nur eine Minderheit (gesamthaft 4\%) setzt mehr als 50\% der Arbeitszeit für die Forschung ein.

Auf der Zielstufe Primarstufe unterscheiden sich die Dozierenden der Erziehungswissenschaft mit $68 \%$ ohne Forschungstätigkeit von ihren Kolleginnen und Kollegen der Mathematik/Mathematikdidaktik (nur 32\% ohne Forschungstätigkeit). Innerhalb des Fachgebiets der Mathematik/Mathematikdidaktik führen die Dozierenden der Sekundarstufe häufiger keine Forschungstätigkeit aus als die Kolleginnen und Kollegen auf der Zielstufe Primarstufe. 
Für die wenigsten der Dozierenden der Deutschschweiz ist Forschung ein Bestandteil ihrer Arbeitstätigkeit. Wenn Dozierende einer Forschungstätigkeit nachgehen, dann häufig in einem Umfang von weniger als 50\%. Nur knappe $4 \%$ der Dozierenden widmen über die Hälfte ihrer Arbeitszeit der Forschung.

Abbildung 5.17: Anteil der Forschungstätigkeit an der Gesamtarbeitszeit der Deutschschweizer Dozierenden dargestellt nach den Fachgruppen und den Zielstufen (gruppiert)

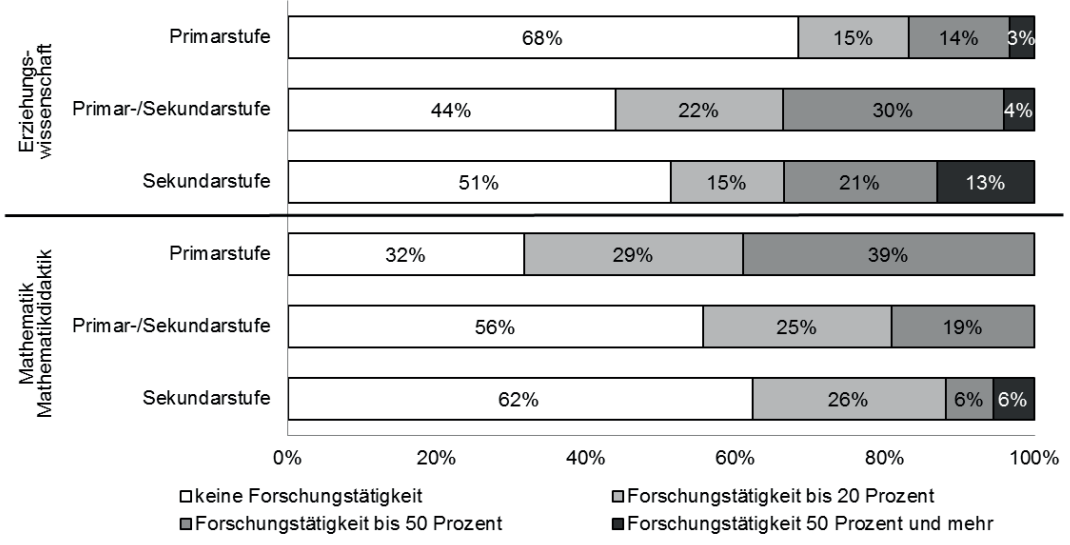

\subsection{Fazit}

Über Dozierende an Lehrerausbildungsinstitutionen ist bis anhin immer noch wenig bekannt. Angesichts der Bedeutung der Dozierenden für eine gelungene Lehrerausbildung überrascht dieser Umstand nach wie vor, insbesondere auch, weil dies seit Jahren immer wieder beklagt wird (Blömeke et al., 2005; Criblez, 2001; Felbrich, Müller \& Blömeke, 2008; Felbrich et al., 2010; Loughran, 1997; Murray \& Male, 2005; Noel, 2006). Die Studie über die Lehrerausbildenden der Deutschschweiz von Criblez (2001) im Rahmen des nationalen Forschungsprogramms 33 zur Wirksamkeit der Lehrerbildungssysteme (Oser \& Oelkers, 2001) fand in den Jahren 1995/96 statt und fiel somit in die Zeit vor der Tertiarisierung der Lehrerausbildung. Mit der hier vorliegenden Studie kann eine Charakterisierung der Dozierenden an den Deutschschweizer Lehrerausbildungsinstitutionen im internationalen Vergleich aufgezeigt wer- 
den, so wie sie sich an den noch jungen Pädagogischen Hochschulen ${ }^{14}$ und der Universität Fribourg ${ }^{15}$ darstellt.

Der männliche Anteil der Dozierenden liegt in der Deutschschweiz bei allen Zielstufen über $50 \%$, in der Zielstufe Primarstufe sind es $57 \%$, bei der gemischten Zielstufe 65\% und bei der Zielstufe Sekundarstufe gar 79\%. Dies ist auch im internationalen Vergleich gesehen ein sehr hoher Anteil an männlichen Dozierenden. Das vorherrschende Phänomen der Volksschule, dass auf höheren Unterrichtsstufen mehr männliche Lehrpersonen tätig sind, lässt sich also auch bei den Lehrerausbildenden beobachten. Die Feminisierung des Lehrerberufes, wie sie in der Volksschule beobachtet werden kann, findet in der Deutschschweizer Lehrerbildung (noch) keinen Niederschlag. Vergleicht man dies mit den Zahlen von 1995/96 (Criblez, 2001), kann, unter Vorbehalt der unterschiedlichen Stichproben, nur eine leichte Zunahme des weiblichen Anteils auf der Sekundarstufe I festgehalten werden, bei einer sonst relativ konstant gebliebenen Verteilung.

Die formalen Ausbildungsqualifikationen setzen sich aus der akademischen Vorbildung und der schulischen Lehrbefähigung zusammen. Die Deutschschweizer Dozierenden haben im internationalen Vergleich einen eher geringen Anteil an promovierten und habilitierten Dozierenden, was vermutlich auf die erst kürzlich erfolgte Tertiarisierung der Lehrerbildung zurückzuführen ist. Auch wurden teilweise Dozierende der ehemaligen Seminare übernommen, deren Nachqualifizierung bisher noch nicht abgeschlossen ist (Annen et al., 2010). Besonders überraschend ist die ausserordentlich tiefe Qualifikation der Deutschschweizer Dozierenden, welche ausschliesslich auf der Zielstufe Sekundarstufe unterrichten. Nur 58\% von ihnen verfügen mindestens über einen universitären Hochschulabschluss (also mindestens Lizentiat oder Master). Wenn man bedenkt, dass im internationalen Vergleich der nächst kleinere Anteil 73\% umfasst, wird noch deutlicher, wie gering der Anteil an universitären Abschlüssen bei der Sekundarstufe in der Deutschschweiz ist. Dies bedeutet auch, dass 42\% der Dozierenden, welche ausschliesslich auf der Sekundarstufe unterrichten, die Anforderungen der EDK (1999a) nach einem Hochschulabschluss nicht erfüllen.

Geschlechtsspezifische Unterschiede der Dozierenden bezüglich der akademischen Qualifikation bestehen, anders als bei Criblez (2001) festgehalten, nicht mehr zu Ungunsten der Frauen. Das Gegenteil ist sogar der Fall: Die Dozentinnen haben tendenziell häufiger einen universitären Abschluss als ihre männlichen Kollegen.

14 Der Start der Pädagogischen Hochschulen mit den neuen Studiengängen erfolgte zu unterschiedlichen Zeitpunkten. In Bern und Wallis starteten die Pädagogischen Hochschulen bereits 2001, in Luzern 2003 und in Basel beispielsweise erst 2004 (Criblez, 2010).

15 Die Universität Fribourg ist die einzige deutschsprachige Universität, welche Lehrpersonen für die Sekundarstufe I ausbildet. Deshalb befindet sich nebst den Pädagogischen Hochschulen auch die Universität Fribourg in der Stichprobe. 
Der Anteil der Dozierenden mit einem Lehrdiplom ist in der Deutschschweiz über alle drei Zielstufen hinweg mit einem Mindestanteil von $89 \%$ sehr hoch. ${ }^{16}$ Im internationalen Vergleich sticht insbesondere der hohe Anteil von $97 \%$ bei der Zielstufe Sekundarstufe hervor. Ein beträchtlicher Anteil von Dozierenden weist sogar mehrere Lehrdiplome auf. Eine deutliche Mehrheit (zwischen 62 und 83\%) haben ein der Zielstufe entsprechendes Lehrdiplom. Die Anforderungen der EDK dürften wesentlich zu diesem hohen Prozentsatz an Lehrdiplomen beitragen.

Trotz der nicht vorhandenen spezifischen Ausbildung für Lehrerausbildende gibt eine Mehrheit an, eine entsprechende Ausbildung absolviert zu haben. Auch bei genauerer Betrachtung der Daten kann nicht entschlüsselt werden, ob die Dozierenden die Antworten entsprechend ihrer formalen Qualifikation oder aufgrund des eigenen Gefühls gegeben haben.

Der Erfahrungshintergrund der Dozierenden wird einerseits über Lehrund anderseits über die Forschungserfahrung beschrieben. Für die Sicherstellung von praktischen Kenntnissen in Bezug auf den Lehrerberuf weisen die Deutschschweizer Dozierenden häufig nicht nur ein Lehrdiplom nach, sondern verfügen erfreulicherweise auch über die praktische Unterrichtserfahrung auf der Zielstufe der Studierenden wie auch auf anderen Stufen. Alle Dozierende der Sekundarstufe verfügen über praktische Erfahrungen in Klassenzimmern. Möglicherweise verfügen die wenigen Dozierenden auf der Primarstufe, die keine Lehrerfahrung auf der Primar- und Sekundarstufe aufweisen, über Erfahrungen auf der Kindergartenstufe. Da diese Parameter in TEDS-M leider vernachlässigt wurden, können dazu keine Ergebnisse präsentiert werden. Die grosse Mehrheit der Dozierenden erfüllt somit die Forderung nach praktischer Erfahrung. Die geforderte akademische Qualifikation der Dozierenden führt, wie oft befürchtet, demnach nicht zu einem Mangel an praktischen Erfahrungen. Es kann also keine Rede davon sein, dass an Pädagogischen Hochschulen Dozierende lehren würden, welche keine eigenen praktischen Erfahrungen im Feld gemacht hätten.

Nebst der praktischen Erfahrung im Klassenzimmer ist gerade hinsichtlich der Tertiarisierung auch die Forschungserfahrung zur Charakterisierung der Lehrerausbildenden wichtig. Die Mehrheit der Dozierenden hat in ihrer Laufbahn eigene Forschungserfahrungen gesammelt. Im internationalen Vergleich sind die Werte der Deutschschweiz jedoch tief. Nur die deutschen Dozierenden der Primarstufe haben noch weniger eigene Forschungserfahrungen. Vergleicht man diese Ergebnisse mit dem Anliegen der Akademisierung und stärkeren Forschungsausrichtung der Lehrerbildung, insbesondere der Pädagogischen Hochschulen, wird deutlich, dass der Prozess der Wissenschafts- und Forschungsorientierung noch nicht die gesamte Dozentenschaft erfasst hat. Die

16 Der Anteil dürfte mit grösster Wahrscheinlichkeit bezüglich der Zielstufe Primarstufe und der Zielstufe Primar- und Sekundarstufe noch höher liegen als hier dargestellt, da in der Erhebung die Anzahl Kindergartenlehrdiplome leider nicht beachtet wurden. 
Wissenschaftsorientierung scheint dadurch weiterhin zu häufig nur ,rezeptiv im Sinne von Wissensreproduktion und nicht produktiv durch eigene Forschung“ (Criblez, 2010; Larcher Klee \& Sieber, 2007) umgesetzt zu werden. Nicht auszublenden ist der Befund, dass Länder, in denen die Studierenden bessere Leistungen in Mathematik und Mathematikdidaktik erreichen, signifikant mehr Dozierende mit Forschungserfahrungen arbeiten, als bei uns in der Deutschschweiz. Daraus könnte die Schlussfolgerung gezogen werden, dass die Umsetzung der EDK-Resolution über die Forschungsverbindlichkeit an Pädagogischen Hochschulen, wenn sie durchgeführt wird, einen positiven Effekt auf die Leistungen der Studierenden haben könnte.

Eine weitere Frage, der sich TEDS-M widmet, ist das Tätigkeitsprofil der Dozierenden. Erhoben wurde der Anteil an der Gesamtarbeitszeit, welche die Dozierenden für Lehre, Forschung, Verwaltung, Dienstleistung und Sonstigem aufwenden. Aus dieser Analyse ist zu folgern, dass die Deutschschweizer Dozierenden hauptsächlich unterrichten, wenig forschen und relativ viel Zeit für Verwaltungsaufgaben, Dienstleistungen und andere Tätigkeiten aufwenden.

In der Deutschschweiz arbeiten ungefähr gleich viele Dozierende mit kleinen, mittleren, grossen und sehr grossen Lehranteilen. Bezüglich der Forschungstätigkeit kann festgestellt werden, dass die meisten Dozierenden keine Zeit für die aktive Wissenschaftsarbeit aufwenden. Nur eine verschwindende Minderheit von 4\% arbeitet mehr als 50\% der Gesamtarbeitszeit in der Forschung. Wenige Deutschschweizer Dozierende können somit mit eigenständigen Beiträgen zur scientific community, z.B. in Form von systematischen Auswertungen von Fachliteratur, dem Generieren von Daten, Auswerten und Publizieren, wie dies von Lehrerausbildenden erwartet werden könnte (Altrichter \& Mayer, 2004), beitragen. Ob angesichts dieser Resultate von einer erfolgreichen Umsetzung des Verwissenschaftlichungsprozesses an den Pädagogischen Hochschulen gesprochen werden kann, erscheint fraglich. Dieser Prozess kann nur durch „Strukturaufbau der Forschung und Entwicklung, der Verknüpfung von Forschung und Lehre sowie der Qualifikationsanforderungen“ (Criblez, 2010) vorangetrieben werden. Beim Betrachten der Tätigkeitsprofile und den Erfahrungen der Dozierenden ist dieser Prozess noch nicht ersichtlich. Natürlich muss mitbeachtet werden, dass die Aufgabenfelder in der Lehrerausbildung zahlreich und vielfältig sind, welche unmöglich alle von einer Person gleichermassen wahrgenommen werden können. Blömeke, Hascher und Mayer (2005) weisen darauf hin, dass sich vollberufliche Lehrerausbildende aber wahrscheinlich kaum aus einem Teilbereich vollständig zurückziehen können. In der Deutschschweiz sind die Pädagogischen Hochschulen zudem so ausgerichtet, dass ein deutlich geringerer Budgetanteil für die Forschung als für die Lehre vorgesehen ist. Wenn alle Dozierenden einen Forschungsanteil erfüllen müssten, könnte dies dazu führen, dass mit einem Minipensum auch nur Mini-Forschung betrieben werden könnte (Gefahr der „Micky-Maus-Forschung“ (Weber, 2008)), welche den Anforderungen einer international anerkannten Forschung nicht standhalten würde. Ein möglicher 
Ausweg besteht darin, dass Dozierende mit verschiedenen Schwerpunkten im Team eng zusammenarbeiten und so von den jeweiligen Stärken (Tätigkeitsprofilen) der anderen profitieren könnten. Eine enge Anbindung der Forschungsabteilung an die Lehre könnte eine Verbindung zwischen der aktuellen Wissenschaftsdiskussion und der Lehre erleichtern und so die Gefahr mindern, dass ein Teil der Dozentenschaft vom aktuellen Wissenschaftsdiskurs ausgeschlossen würde.

\subsection{Literatur}

Altrichter, H. \& Mayer, J. (2004). Forschung in der Lehrerbildung. In S. Blömeke, P. Reinhold, G. Tulodziecki \& J. Wildt (Hrsg.), Handbuch Lehrerbildung (S. 636). Bad Heilbrunn: Klinkhardt.

Ambühl, H. \& Stadelmann, W. (2010). Tertiarisierung der Lehrerinnen- und Lehrerbildung: Bilanztagung I. Bern: EDK.

Annen, L., Cattaneo, M. A., Denzler, S., Diem, A., Grossenbacher, S., Hof, S., Kull, M., Vögeli-Mantovani, U. \& Wolter, S.C. (2010). Bildungsbericht Schweiz 2010. Aarau: SKBF.

Annen, L., Cattaneo, M.A., Denzler, S., Diem, A., Grossenbacher, S., Hof, S., Berry, A. \& Loughran, J. (2002). Developing an understanding of learning fo teach in teacher education. In J. Loughran \& T. Russell (Hrsg.), Improving teacher education practices through self-study (S. 13-29). London: Routledge.

Blömeke, S., Hascher, T. \& Mayr, J. (2005). Beruf: LehrerbildnerIn. Selbstverständnis, Aufgaben, Qualifizierung. Journal für LehrerInnenbildung, 5(4), $7-20$.

Blömeke, S., Kaiser, G. \& Lehmann, R. H. (2008). Professionelle Kompetenz angehender Lehrerinnen und Lehrer. Wissen, Überzeugungen und Lerngelegenheiten deutscher Mathematikstudierender und -referendare. Erste Ergebnisse zur Wirksamkeit der Lehrerausbildung. Münster: Waxmann.

Blume, R. (1971). Humanizing teacher education. PHI Delta Kappan, 53, 411-415.

COHEP (2007). Strategie COHEP 2007-2011. Bern: Cohep.

Criblez, L. (1994). Lehrerbildung in der Schweiz: Vielfalt ohne Koordination? Bildungsforschung und Bildungspraxis, 16, 139-160.

Criblez, L. (2001). Die Ausbildnerinnen und Ausbildner - Hauptakteure der Lehrerbildung. Ausgewählte Ergebnisse aus der Befragung der Ausbildnerinnen und Ausbildner. In F. Oser \& J. Oelkers (Hrsg.), Die Wirksamkeit der Lehrerbildungssysteme. Von der Allrounderausbildung zur Ausbildung professioneller Standards (S. 437-494). Zürich: Rüegger. 
Criblez, L. (2010). Die Reform der Lehrerinnen- und Lehrerbildung in der Schweiz seit 1990: Reformprozesse, erste Bilanz und Desiderata. In H. Ambühl \& W. Stadelmann (Hrsg.), Tertiarisierung der Lehrerinnen- und Lehrerbildung: Bilanztagung I (S. 22-58). Bern: EDK.

Darling-Hammond, L. (2000). How Teacher Education Matters. Journal of Teacher Education, 51(3), 166-173.

Eckert, T. (2006). Die Feminisierung der Lehrerschaft als Kohortenphänomen -Entwicklungen der Lehrerschaft an allgemein bildenden Schulen BadenWürttembergs. Bildungsforschung, 3(1), 19.

EDK (1999a). Reglement über die Anerkennung von Hochschuldiplomen für Lehrkräfte der Sekundarstufe I vom 26. August 1999. Bern: EDK.

EDK (1999b). Reglement über die Anerkennung von Hochschuldiplomen für Lehrkräfte für Lehrkräfte der Vorschulstufe und der Primarstufe vom 10. Juni 1999. Bern: EDK.

EDK (2007). Lehrerinnen und Lehrer und anderes Personal im Bildungsbereich. Online unter: http://www.edk.ch/dyn/bin/12961-13437-1-eurydice_08d.pdf (4.10.2012).

EDK (2008). Anschlussbericht zum Masterplan Pädagogische Hochschulen. Bern: EDK.

Felbrich, A., Müller, C. \& Blömeke, S. (2008). Lehrerausbildnerinnen und Lehrerausbildner der ersten und zweiten Phase. In S. Blömeke, G. Kaiser \& R. H. Lehmann (Hrsg.), Professionelle Kompetenz angehender Lehrerinnen und Lehrer. Wissen, Überzeugungen und Lerngelegenheiten deutscher Mathematikstudierender und -referendare. Erste Ergebnisse zur Wirksamkeit der Lehrerausbildung (S. 363-390). Münster: Waxmann.

Felbrich, A., Schmotz, C., Kaiser, G., Hacke, S. \& Lehmann, R. H. (2010). Merkmale von Lehrerausbildenden für die Primarstufe im internationalen Vergleich. In S. Blömeke, G. Kaiser \& R. H. Lehmann (Hrsg.), Professionelle Kompetenz und Lerngelegenheiten angehender Sekundarlehrkräfte im internationalen Vergleich (S. 73-96). Münster: Waxmann.

Furger, M. (2010). Das Bildungskartell. NZZ am Sonntag, 7. November.

Guggenbühl, A. (2001). Böse Buben. NZZ Folio, 8, 56-58.

Herzog, W. (1999). Professionalisierung im Dilemma. Braucht die Lehrerinnen- und Lehrerbildung eine eigene Wissenschaft? Beiträge zur Lehrerbildung , 17(3), 340-374.

Hügli, A. (1998). Warum Forschung für die Ausbildung der Lehrerinnen und Lehrer vonnöten ist. Beiträge zur Lehrerbildung, 16(1), 5-17.

Korthagen, F., Loughran, J. \& Lunenberg, M. (2005). Teaching teachers--studies into the expertise of teacher educators: an introduction to this theme issue. Teaching and Teacher Education, 21(2), 107-115.

Koster, B., Brekelmans, M., Korthagen, F. \& Wubbels, T. (2005). Quality requirements for teacher educators. Teaching and Teacher Education, 21(2), $157-176$. 
Koster, B. \& Dengerink, J. (2001). Towards a Professional Standard for Dutch Teacher Educators. European Journal of Teacher Education, 24(3), 343-354.

Lanier, J. E. \& Little, J. W. (1986). Research on teacher education. In M. C. Wittrock (Hrsg.). Handbook of research on teaching (S. 527-569). New York: Macmillan.

Larcher Klee, S. \& Sieber, P. (2007). Wissen und Wissensproduktion in der Lehrerinnen- und Lehrerbildung. In W. Bircher, S. Larcher Klee, M. Schmid \& P. Sieber (Hrsg.), Der Weg zur Pädagogischen Hochschule (S. 101-110). Zürich: Pestalozzianum.

Lehmann, L., Criblez, L., Guldimann, T., Fuchs, W. \& Périsset Bagnoud, D. (2007). Lehrerinnen- und Lehrerbildung in der Schweiz. Bericht im Rahmen der Bildungsberichterstattung 2006. Aarau: Schweizerische Koordinationsstelle für Bildungsforschung.

Loughran, J. (1997). Teaching about teaching: Principles and practice. In J. Loughran \& T. Russell (Hrsg.), Teaching about teaching: Purpose, passion and pedagogy in teacher education (S. 57-70). London, Washington: Falmer Press.

Lunenberg, M., Loughran, J., Schildkamp, K., Beishuizen, J., Meirink, J. \& Zwart, R. (2007). Self-study in a community of learning researchers. What can we do to help teachers/teacher educators benefit from our research? European educational research journal, 6(4), 411-423.

Murray, F. B. (2001). The Overreliance of Accreditors on Consensus Standards. Journal of Teacher Education, 52(3), 211-222.

Murray, J. \& Male, T. (2005). Becoming a teacher educator: evidence from the field. Teaching and Teacher Education, 21(2), 125-142.

Noel, P. (2006). The secret life of teacher educators: becoming a teacher educator in the learning and skills sector. Journal of Vocational Education \& Training, 58(2), 151-170.

Oser, F. \& Oelkers, J. (2001). Die Wirksamkeit der Lehrerbildungssysteme. Von der Allrounderausbildung zur Ausbildung professioneller Standards. Zürich: Rüegger.

Robinson, M. \& McMillan, W. (2006). Who teaches the teachers? Identity, discourse and policy in teacher education. Teaching and Teacher Education, 22(3), 327-336.

Ruchti, B. \& Meier, P. J. (2009). Buben sind die Dummen. Beobachter, 9. Online unter: http://www.beobachter.ch/arbeit-bildung/schule/artikel/schule buden-sind-die-dummen/ (25.3.2015).

Russell, T. (1997). Teaching teachers: How I teach IS the message. In J. Loughran \& T. Russell (Hrsg.), Teaching about teaching: Purpose, passion and pedagogy in teacher education (S. 32-47). London, Washington: Falmer Press.

Schmotz, C., Felbrich, A., Lehmann, R. H., Hacke, S. \& Kaiser, G. (2010). Merkmale von Sekundarstufen-I-Lehrerausbildenden im internationalen 
Vergleich. In S. Blömeke, G. Kaiser \& R. H. Lehmann (Hrsg.), Professionelle Kompetenz und Lerngelegenheiten angehender Sekundarlehrkräfte im internationalen Vergleich (S. 73-96). Münster: Waxmann.

Schön, D. A. (1983). The Reflective Practitioner. How Professionals Think in Action. New York: Basic Books.

Shulman, L. S. (1986). Those who understand: Knowledge growth in teaching. Educational Researcher, 15(2), 4-14.

Smith, K. (2005). Teacher educators' expertise: what do novice teachers and teacher educators say? Teaching and Teacher Education, 21(2), 177-192.

Vogt, F. \& Pilloud, J. (2010). Dozierende. In H. Ambühl \& W. Stadelmann (Hrsg.), Tertiarisierung der Lehrerinnen- und Lehrerbildung: Bilanztagung I (S. 79-80). Bern: EDK.

Weber, S. (2008). Das Google-Copy-Paste-Syndrom. Wie Netzplagiate Ausbildung und Wissen gefährden. Hannover: Heise Zeitschriften Verlag.

Zeichner, K. (2005). Becoming a teacher educator: a personal perspective. Teaching and Teacher Education, 21(2), 117-124. 
